University of New Hampshire

University of New Hampshire Scholars' Repository

\title{
The Energetic Particle Detector (EPD) Investigation and the Energetic Ion Spectrometer (EIS) for the Magnetospheric Multiscale (MMS) Mission
}

B. H. Mauk

Johns Hopkins University

J. B. Blake

Aerospace Corporation

D. N. Baker

University of Colorado Boulder

J. H. Clemmons

Aerospace Corporation

Geoffrey Reeves

Los Alamos National Laboratory

Follow this and additional works at: https://scholars.unh.edu/ssc

Part of the Other Astrophysics and Astronomy Commons

See next page for additional authors

\section{Recommended Citation}

178. Mauk, B. H., J. B. Blake. Daniel Baker, James Clemmons, H. E. Spence, Geoff Reeves, George Ho, Joseph Westlake, A. Jacques, S. E. Jaskulek, C. E. Schlemm, Patrick Hill, L. E. Brown, S. A. Cooper, R. S. Gurnee, C. Hammock, J. R. Hayes, J. C. Hutcheson, D. G. Mitchell, K. S. Nelson, N. Paschalidis, E. Rossano, S. Kerem, M. R. Stokes, The Energetic Particle Detector (EPD) Investigation and the Energetic Ion Spectrometer (EIS) for the Magnetospheric Magnetoscale (MMS) Mission, Space Science Reviews, DOI: 10.1007/s11214-014-0055-5, 2014.

This Article is brought to you for free and open access by the Institute for the Study of Earth, Oceans, and Space (EOS) at University of New Hampshire Scholars' Repository. It has been accepted for inclusion in Space Science Center by an authorized administrator of University of New Hampshire Scholars' Repository. For more information, please contact Scholarly.Communication@unh.edu. 


\section{Authors}

B. H. Mauk, J. B. Blake, D. N. Baker, J. H. Clemmons, Geoffrey Reeves, Harlan E. Spence, S. E. Jaskulek, C. E. Schlemm, L. E. Brown, Steve Cooper, J. V. Craft, J. F. Fennell, R. S. Gurnee, C. M. Hammock, J. R. Hayes, P. A. Hill, G. C. Ho, J. C. Hutcheson, A. D. Jacques, S. Kerem, D. G. Mitchell, K. S. Nelson, N. P. Paschalidis, E. Rossano, M. R. Stokes, and J. H. Westlake 


\title{
The Energetic Particle Detector (EPD) Investigation and the Energetic Ion Spectrometer (EIS) for the Magnetospheric Multiscale (MMS) Mission
}

\author{
B.H. Mauk • J.B. Blake • D.N. Baker • J.H. Clemmons • G.D. Reeves • H.E. Spence • \\ S.E. Jaskulek • C.E. Schlemm • L.E. Brown - S.A. Cooper · J.V. Craft • J.F. Fennell • \\ R.S. Gurnee · C.M. Hammock · J.R. Hayes · P.A. Hill • G.C. Ho · J.C. Hutcheson • \\ A.D. Jacques • S. Kerem • D.G. Mitchell • K.S. Nelson • N.P. Paschalidis • E. Rossano • \\ M.R. Stokes $\cdot$ J.H. Westlake
}

Received: 14 February 2014 / Accepted: 9 June 2014

(C) The Author(s) 2014. This article is published with open access at Springerlink.com

\begin{abstract}
The Energetic Particle Detector (EPD) Investigation is one of 5 fields-andparticles investigations on the Magnetospheric Multiscale (MMS) mission. MMS comprises 4 spacecraft flying in close formation in highly elliptical, near-Earth-equatorial orbits targeting understanding of the fundamental physics of the important physical process called magnetic reconnection using Earth's magnetosphere as a plasma laboratory. EPD comprises two sensor types, the Energetic Ion Spectrometer (EIS) with one instrument on each of the 4 spacecraft, and the Fly's Eye Energetic Particle Spectrometer (FEEPS) with 2 instruments on each of the 4 spacecraft. EIS measures energetic ion energy, angle and elemental compositional distributions from a required low energy limit of $20 \mathrm{keV}$ for protons and $45 \mathrm{keV}$ for oxygen ions, up to $>0.5 \mathrm{MeV}$ (with capabilities to measure up to $>1 \mathrm{MeV}$ ). FEEPS measures instantaneous all sky images of energetic electrons from $25 \mathrm{keV}$ to $>0.5 \mathrm{MeV}$,
\end{abstract}

B.H. Mauk ( $\varangle) \cdot$ S.E. Jaskulek · C.E. Schlemm · L.E. Brown · S.A. Cooper · R.S. Gurnee · J.R. Hayes · P.A. Hill · G.C. Ho · J.C. Hutcheson - S. Kerem · D.G. Mitchell · K.S. Nelson · M.R. Stokes · J.H. Westlake

The Johns Hopkins University, Applied Physics Laboratory, Laurel, MD, USA

e-mail: barry.mauk@jhuapl.edu

J.B. Blake · J.H. Clemmons · J.F. Fennell

Aerospace Corporation, El Segundo, CA, USA

D.N. Baker · J.V. Craft

University of Colorado, LASP, Boulder, CO, USA

H.E. Spence

University of New Hampshire, Durham, NH, USA

G.D. Reeves

Los Alamos National Laboratory, Los Alamos, NM, USA

A.D. Jacques · N.P. Paschalidis · E. Rossano

NASA Goddard Space Flight Center, Greenbelt, MD, USA

C.M. Hammock

NASA Johnson Space Center, Houston, TX, USA

Published online: 28 June 2014 
and also measures total ion energy distributions from $45 \mathrm{keV}$ to $>0.5 \mathrm{MeV}$ to be used in conjunction with EIS to measure all sky ion distributions. In this report we describe the EPD investigation and the details of the EIS sensor. Specifically we describe EPD-level science objectives, the science and measurement requirements, and the challenges that the EPD team had in meeting these requirements. Here we also describe the design and operation of the EIS instruments, their calibrated performances, and the EIS in-flight and ground operations. Blake et al. (The Flys Eye Energetic Particle Spectrometer (FEEPS) contribution to the Energetic Particle Detector (EPD) investigation of the Magnetospheric Magnetoscale (MMS) Mission, this issue) describe the design and operation of the FEEPS instruments, their calibrated performances, and the FEEPS in-flight and ground operations. The MMS spacecraft will launch in early 2015, and over its 2-year mission will provide comprehensive measurements of magnetic reconnection at Earth's magnetopause during the 18 months that comprise orbital phase 1, and magnetic reconnection within Earth's magnetotail during the about 6 months that comprise orbital phase 2 .

Keywords NASA mission · Magnetospheric multiscale $\cdot$ Magnetosphere $\cdot$ Magnetic reconnection $\cdot$ Space plasma $\cdot$ Particle acceleration

\section{EPD Introduction, Background, Science Goals}

\subsection{Background and Overview}

The purpose of NASA's Magnetospheric Multiscale (MMS) mission, as described by Burch et al. (this issue), is to provide understanding of the fundamental physics of the critical energy conversion process of magnetized space plasmas called Magnetic Reconnection. Magnetic reconnection is a spatially localized process that converts magnetic energy that is derived from the flow energy of ionized gases (plasmas), into particle energy in the form of different forms of plasma flow, heating, and particle energization To provide that understanding, the MMS mission comprises 4 spacecraft that fly in formation (10 to $400 \mathrm{~km}$ apart) in highly elliptical orbits $(1.2 \times 12$ to $1.2 \times 25 \mathrm{RE})$, thereby obtaining simultaneous, multipoint measurements of known reconnection sites on the dayside on Earth's magnetopause (the boundary of Earth's magnetosphere near $12 \mathrm{RE}$ on the dayside) and within Earth's comet-like magnetic tail on the nightside (with reconnection sites maximizing in occurrence near $25 \mathrm{RE}$ ). Each MMS spacecraft hosts a comprehensive array of particles and fields instruments that make localized in situ measurements of electron and ion energy, directional, and species distributions from low to high energies; the time and spatially varying electric and magnetic field vectors; and the electric and magnetic fields of the waves that propagate within the plasmas.

The Energetic Particle Detector (EPD) investigation contributes to these measurements by measuring the high energy portions of the electron and ion energy, directional, and compositional distributions. On each spacecraft EPD includes an Energetic Ion Spectrometer (EIS) and a pair of all-sky particle samplers called the Fly's Eye Energetic Particle Sensor (FEEPS). The FEEPS instruments use multiplicities of solid state detector (SSD) sensors to maximize the number of viewing directions. The EIS instruments use microchannel plate (MCPs) and SSDs, configured to measure ion times-of-flight (TOF) and particle energies to obtain clean measurements of ion spectra and ion composition for 6 different views within a plane. Schematics of these two sensor types, along the organization of the highly experienced individuals that comprise the EPD team are shown in Fig. 1. Figure 2 provides 


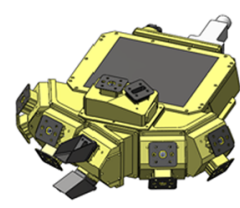

FEEPS

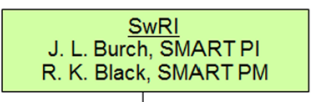

JHU/APL

B. H. Mauk EPD Lead, Co-I P. Hill, EPD PM

G. Ho, EIS Inst. Sci.

J. Westlake, EPD Instr. Sci.

C. Schlemm, EIS Lead Eng.

S. Jaskulek, EPD Sys. Eng.

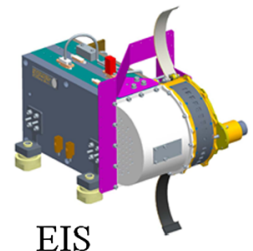

EIS
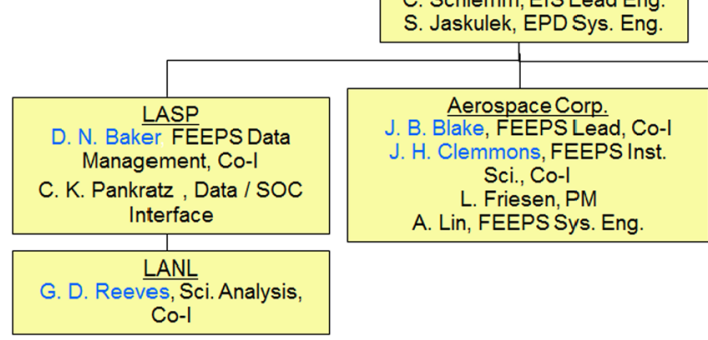

University of New Hampshire

H. E. Spence, EPD Cal., Co-I

A. Lin, FEEPS Sys. Eng.

\section{SwRI Management \\ EPD Team \\ Co-Investigators}

Fig. 1 The MMS Energetic Particle Detector (EPD) Investigation science team with schematics of the two different EPD sensor types

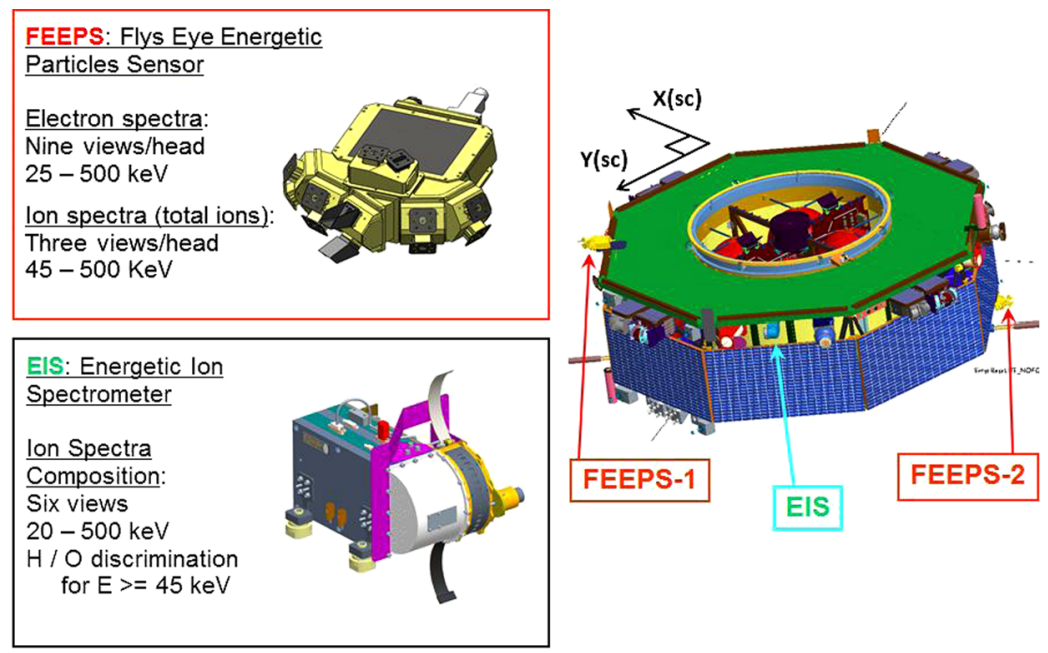

Fig. 2 Capability summaries and mounting positions of the two EPD instrument types

summary information about the capabilities of the two sensor types, and shows where the sensors are placed on each MMS spacecraft. These instruments together measure: (1) the energy-angle distribution and composition of ions $(20$ to $500 \mathrm{keV}$, with a goal of 10 to $1000 \mathrm{keV}$ ) at a time resolution of $<30$ seconds, (2) the energy-angle distribution of total ions (45-500 keV, with a goal of 40-1000 keV) at a time resolution of $<10$ seconds, and (3) the coarse and fine energy-angle distribution of energetic electrons (25-500 keV, with a goal of $25-1000 \mathrm{keV}$ ) at time resolutions of $<0.5$ and $<10$ seconds, respectively.

In this paper we present a high level overview of the EPD (EIS + FEEPS) science objectives, requirements, challenges, and hardware configuration. The paper then goes on to provide details of the EIS instruments. The details of the FEEPS instruments are provided in a companion paper (Blake et al. this issue). EIS has substantial similarities to the Jupiter 


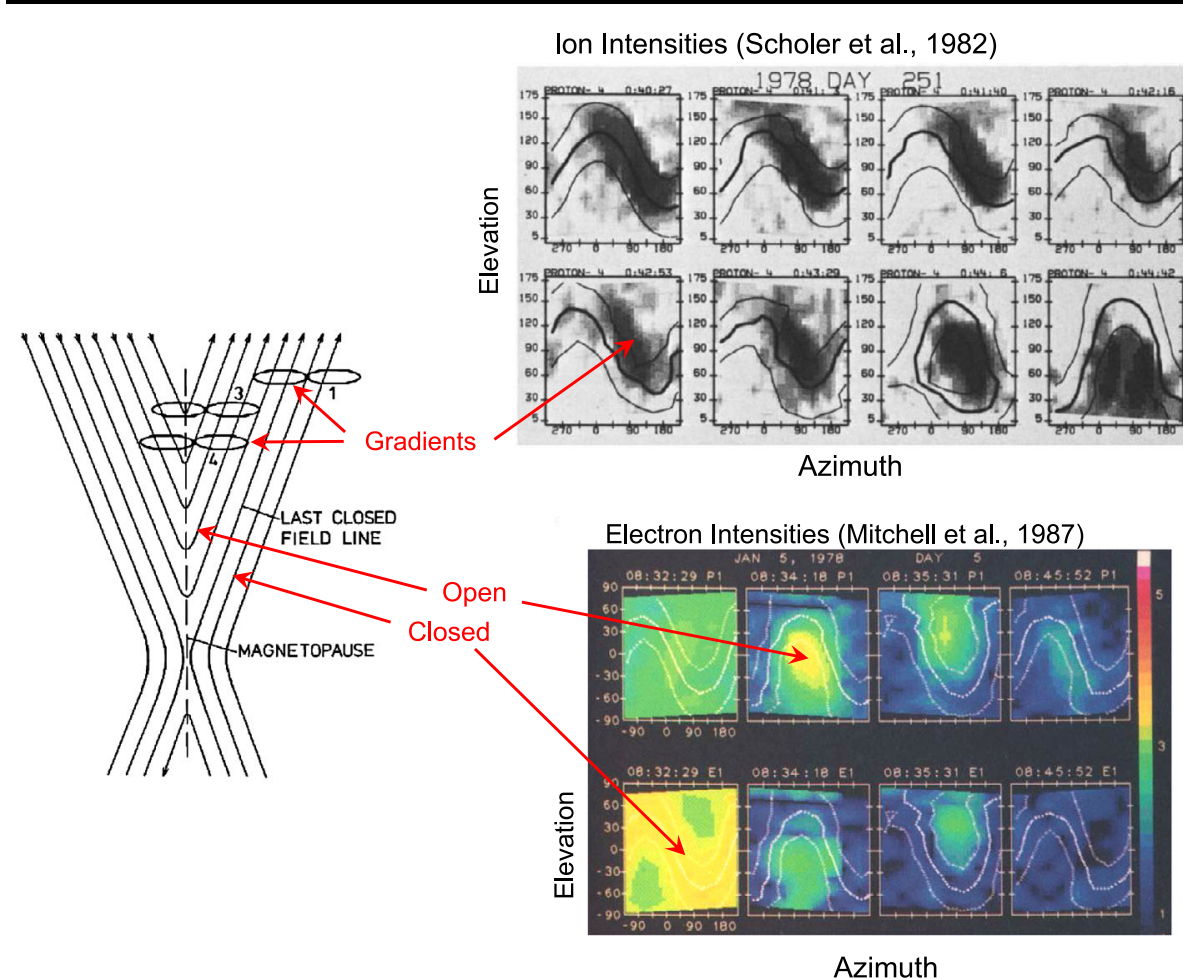

Fig. 3 Illustration of the power of energetic ion and electron measurements in diagnosing the geometric and topological structure of reconnection sites (top and left panels from Scholer et al. 1982; bottom panel from Mitchell et al. 1987). The dark (white) lines in the top (bottom) panels are contours of constant pitch angle

Energetic Particle Detector Instrument (JEDI) now on its way to Jupiter with the Juno mission (Mauk et al. 2013) and the RBSPICE instrument now flying on the twin Van Allen Probes mission (Mitchell et al. 2013). We will referring to these sister instruments, and the respective overview papers, a number of times within the present paper.

\subsection{EPD Science Objectives}

The Energetic Particle Detector suite and investigation supports the study of the fundamental physics of magnetic reconnection by:

(1) Remotely sensing the positions and speeds of boundaries and other structures near reconnection sites using energetic ions.

(2) Sensing the magnetic topology of near reconnection sites using energetic electrons.

(3) Remotely sensing reconnection acceleration sites.

(4) Determining the cause of energization of energetic electrons and ions by reconnection.

\subsection{Scientific Context and Background}

Figure 3 shows how EPD Objective \#1 is achieved. It shows that energetic ion angular distributions (upper right) can be used to remotely sense the structure of near reconnection sites. This remote sensing is made possible by the large radii of gyration (circles in the left 


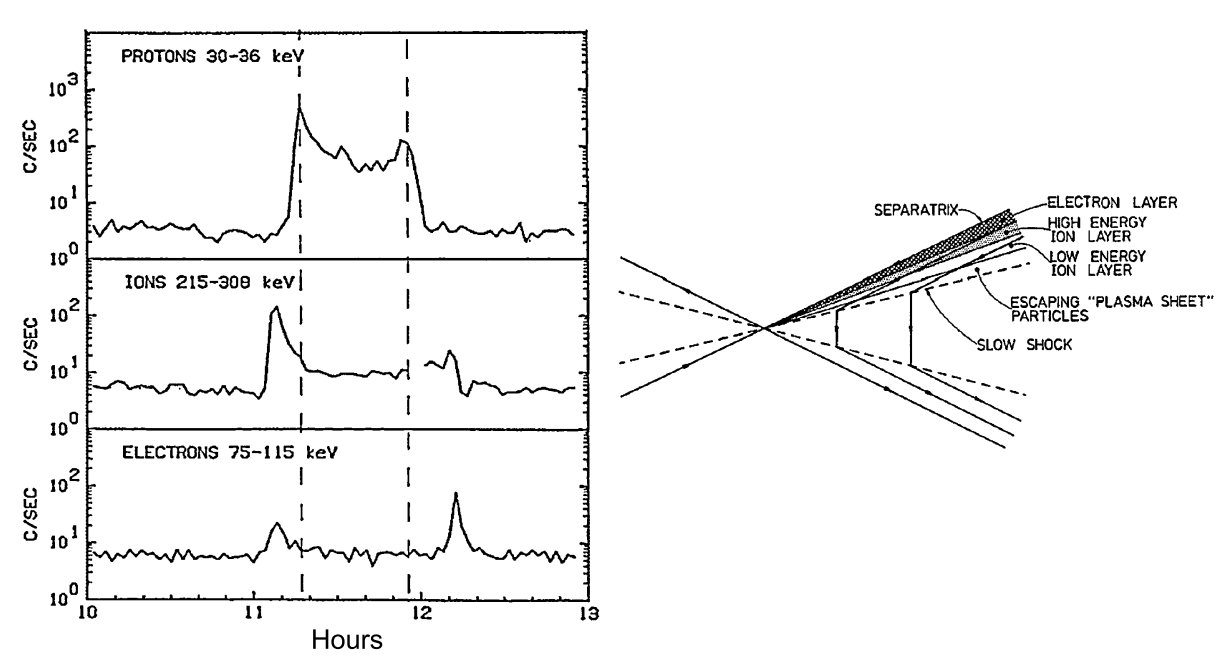

Fig. 4 Illustration of the use of energetic particles to diagnose distant acceleration by, what is thought to be, magnetic reconnection. From Scholer et al. 1987

panel) of the energetic ions measured by EPD. High energy ions are required because they are not substantially affected by the strong electric fields in the vicinity of reconnection sites.

Figure 3 also shows how EPD Objective \#2 is achieved. It shows that the symmetry of energetic electron angular distributions (lower right) reveal whether magnetic field lines are open (one end connected to Earth's magnetic field and one end connected to the interplanetary environment) or closed (both ends connected to Earth). The openness or closeness of magnetic field lines is a critical factor in understanding the configuration of the magnetic reconnection sites. The high energy electrons are needed because they not substantially affected by the strong electric fields in the vicinity of reconnection sites.

The remote sensing of reconnection sites (Objective \#3) is illustrated in Fig. 4. Here the velocity filtering of energetic electrons and ions, engendered by the strong reconnectioninduced flows, allows us to infer the occurrence of localized acceleration or access sites that have been interpreted as arising from localized reconnection. Observations like that shown in Fig. 4 have over many years led to the inference that reconnection can energize electrons and ions to relatively high energy (10's to 100's of keV and higher), even while theorists struggle to understand how (or even if) reconnection sites can retain energetic particles long enough to impart these kinds of energies to them.

The most scientific compelling of the EPD objectives is \#4, the determination of the existence of and causes of high energy acceleration by reconnection processes. High energy electron acceleration by reconnection has been inferred by many observations (e.g. x-ray emissions from solar flares, Lin and Hudson 1971), but a particularly compelling observation is that of Øieroset et al. (2002), where acceleration of electrons to $>300 \mathrm{keV}$ was observed centered on electromagnetic parameters indicative of a passage close to a reconnection X-line. Transient acceleration events have long been observed in the tail in association with substorm processes, and such processes have over the years been thought to be regulated by reconnection (e.g. Baker et al. 1996; 2002). How this acceleration occurs, and specifically how the reconnection site can retain the electrons long enough, is highly controversial, with a number of different models invoked to explain the observations. Drake et al. $(2003 ; 2005)$ proposed that electrons are energized through a Fermi-like process as 
Fig. 5 Comparison between a model of electron acceleration associated with magnetic reconnection in Earth's magnetotail (top and middle panels), and observations made by the Cluster spacecraft (bottom panels) (Egedal et al. 2010)
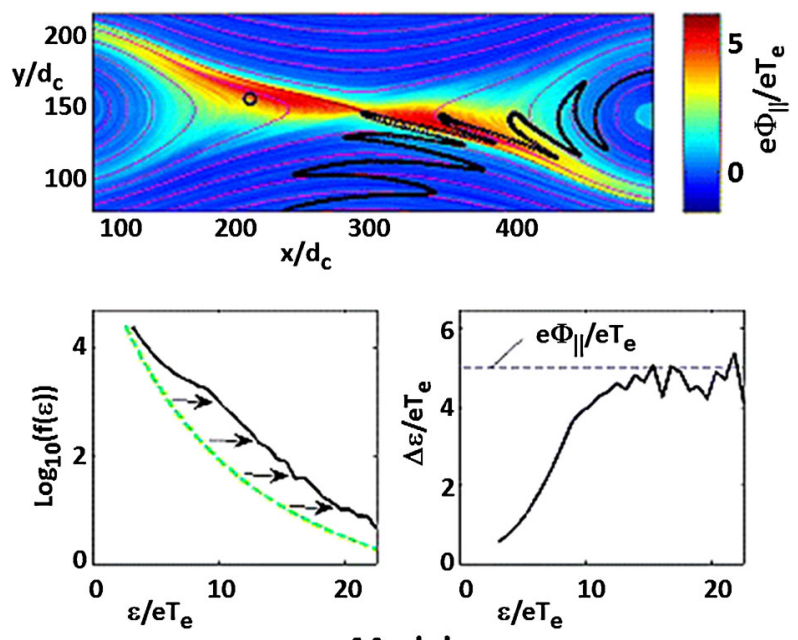

Model

Cluster Observations
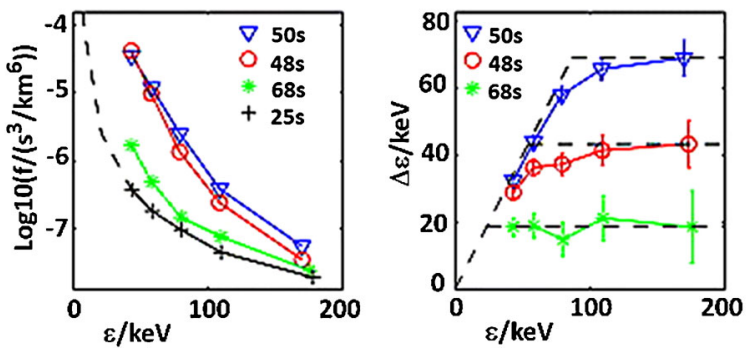

electron populations are compressed within collapsing plasma bubbles that arise from the reconnection process. Pritchett (2005) proposed that electrons can be retained in the vicinity of the reconnection $\mathrm{x}$-line with a guide field long enough for the electrons to be acted upon over large distances. Hoshino (2005) proposed a multi-step energization process, where energization is achieved through a combination of what he calls electron "surfing" on evolving electric field structures, followed by "Speiser-Meander" acceleration, and then followed by betatron acceleration associated with outflow-induced compression. The idea of a direct action by large scale electric field has been given substantial support by the comparisons between modeling and Cluster observations in Egedal et al. (2010). Here (Fig. 5), a specific predicted signature of modeled acceleration by reconnection (middle) is found to nicely reproduce signatures observed near reconnection sites by Cluster energetic electron sensors (bottom). A critical challenge of MMS and EPD is to determine which of these or other mechanisms are responsible for the electron acceleration.

Much more controversial is the hypothesized role of reconnection in the acceleration of ions. While many historical observations like that shown in Fig. 4 have been used to infer the acceleration of ions by localized reconnection, a well-recognized problem is that the relatively small scale reconnection sites theoretically have difficulty in retaining the ions, with their large gyro-radii, long enough to impart substantial amounts of energy to them. The literature is very sparse in relation to reconnection theoretical models that can accelerate ions to 10 's and $>100 \mathrm{keV}$. Grigorenko et al. $(2009 ; 2011)$ have suggested that the conditioning of the neutral sheet within the reconnection exhaust region, but very close to the 

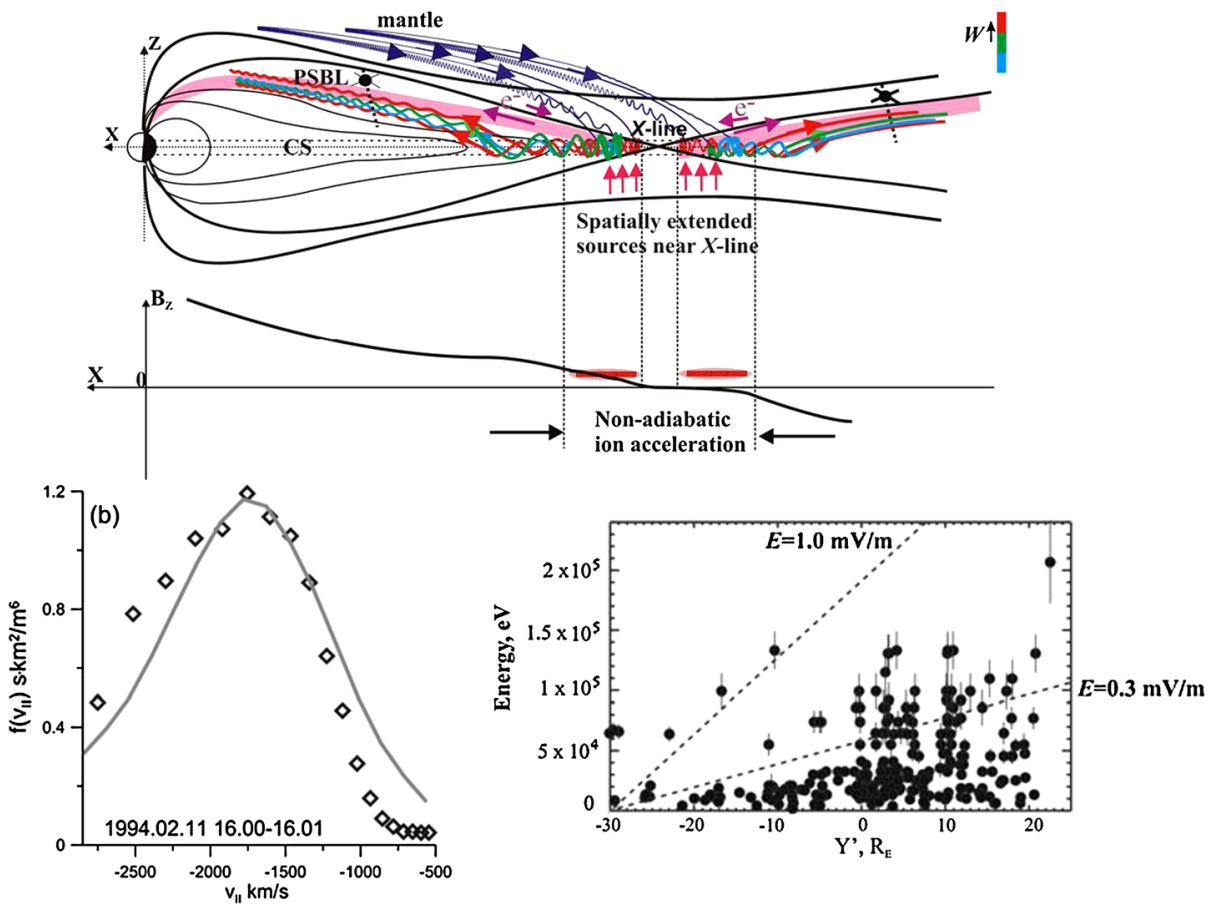

Fig. 6 Schematic (top), an observation versus modeling study of a single event (lower left), and a statistical study (lower right) of the acceleration of energetic ion beams associated with magnetic reconnection in Earth's magnetotail as measured by Geotail (from Grigorenko et al., 2009; 2011)

reconnection site as diagnosed by energetic electron distribution symmetries, can explain the occurrences of ion acceleration observed by the Geotail mission to 10 's of keV and occasionally to energies substantially greater than $100 \mathrm{keV}$ (Fig. 6). Alternatively and perhaps for higher energies, reconnection could act as a gate for the release of energetic ions trapped within a reservoir. Perhaps processes that are stimulated by reconnection, that is: secondary processes, are responsible for the acceleration? With regard to secondary processes, much attention has recently been paid to traveling depolarization fronts that are thought to be launched by reconnection (Runov et al. 2009; Sitnov et al. 2009; Angelopoulos et al. 2013; Birn et al. 2013). These fronts offer a secondary site of possible ion acceleration that could explain observed burst-like energetic ion events. Again, it is a critical challenge to determine which of these or other mechanisms are responsible for the observed transient energetic ion features within Earth's magnetotail that have in the past been attributed to reconnection.

Table 1 provides a list of some of the fundamental acceleration mechanisms that have appeared in the literature. An important element in discriminating between ion acceleration processes is ion composition. The comments provided in the table provide a qualitative assessment as to the sensitivity of the mechanism to the mass of the particle involved.

\section{EPD and EIS Requirements}

Figure 7 shows where the EPD requirements reside within a high level representation of the MMS Program Level (Level-1) requirements. Table 2 shows the flow down of the EPD 
Table 1 Acceleration mechanism and its relation to mass composition

\begin{tabular}{|c|c|c|}
\hline Mechanism & Sample references & Comments \\
\hline Slow mode shock acceleration & Sato et al. 1982 & $\begin{array}{l}\text { Ions favored, pick-up or } \\
\text { Fermi-like: Highly mass } \\
\text { sensitive }\end{array}$ \\
\hline $\mathrm{X}$-line linear acceleration & $\begin{array}{l}\text { Pellinen and Heikkila 1978; } \\
\text { Sato et al. 1982; } \\
\text { Kuznetsova et al. 1996; Pritchett 2005; } \\
\text { Egedal et al. } 2010\end{array}$ & $\begin{array}{l}\text { Electrons and ions: Ion mass } \\
\text { sensitivity related to gyro-radii } \\
\text { and size of demagnetization } \\
\text { zone }\end{array}$ \\
\hline $\begin{array}{l}\text { Betatron acceleration in } \\
\text { evolving magnetic fields }\end{array}$ & $\begin{array}{l}\text { Pellinen and Heikkila 1978; Hoshino } \\
\text { 2005; Birn and Hesse 1994. Birn et al. } \\
\text { 2013. }\end{array}$ & $\begin{array}{l}\text { Electrons and ions: Ion mass } \\
\text { sensitivity related to gyro-radii } \\
\text { and size of demagnetization } \\
\text { zone }\end{array}$ \\
\hline $\begin{array}{l}\text { Speiser (stationary and time } \\
\text { dependent) }\end{array}$ & $\begin{array}{l}\text { Fujimoto and Nakamura 1994; } \\
\text { Sachsenweger et al. 1989; Grigorenko et } \\
\text { al. 2009; 2011. Birn and Hesse } 1994 .\end{array}$ & $\begin{array}{l}\text { Electrons and Ion; Ions } \\
\text { favored: Highly mass sensitive }\end{array}$ \\
\hline $\begin{array}{l}\text { Fermi acceleration: Coherent } \\
\text { and diffusive }\end{array}$ & $\begin{array}{l}\text { Kuznetsova et al. 1996; } \\
\text { Owen and Mist 2001; } \\
\text { Birn and Hesse } 1994\end{array}$ & $\begin{array}{l}\text { Electrons and Ions; } \\
\text { Non-adiabatic limits favor ions } \\
\text { in a highly mass sensitive } \\
\text { fashion. }\end{array}$ \\
\hline $\begin{array}{l}\text { "Pick-up": Transient } \\
\text { Fermi-like (aligned) and } \\
\text { gyro-like (perpendicular) }\end{array}$ & $\begin{array}{l}\text { Browning and Vekstein 2001; } \\
\text { Hoshino et al. 2001; } \\
\text { Delcourt et al. 1990; Mauk 1986; 1989; } \\
\text { Nosé et al. 2000; Sánchez et al. } 1993 .\end{array}$ & $\begin{array}{l}\text { Ions favored: Highly mass } \\
\text { sensitive }\end{array}$ \\
\hline $\begin{array}{l}\text { Turbulent confinement and } \\
\text { reflection }\end{array}$ & $\begin{array}{l}\text { Matthaeus et al. 1984; } \\
\text { Owen and Mist } 2001\end{array}$ & $\begin{array}{l}\text { Electrons and ions: Ion mass } \\
\text { sensitivity related to geometry } \\
\text { of reconnection site and to } \\
\text { gyro-radii }\end{array}$ \\
\hline $\begin{array}{l}\text { Buneman electron holes (A } \\
\text { Fermi process) }\end{array}$ & $\begin{array}{l}\text { Øieroset et al. 2002; after Drake et al. } \\
2003\end{array}$ & Electrons \\
\hline
\end{tabular}

Fig. 7 High level display of MMS Program Level (Level-1) Measurement requirements identifying those requirements levied on the EPD Investigation

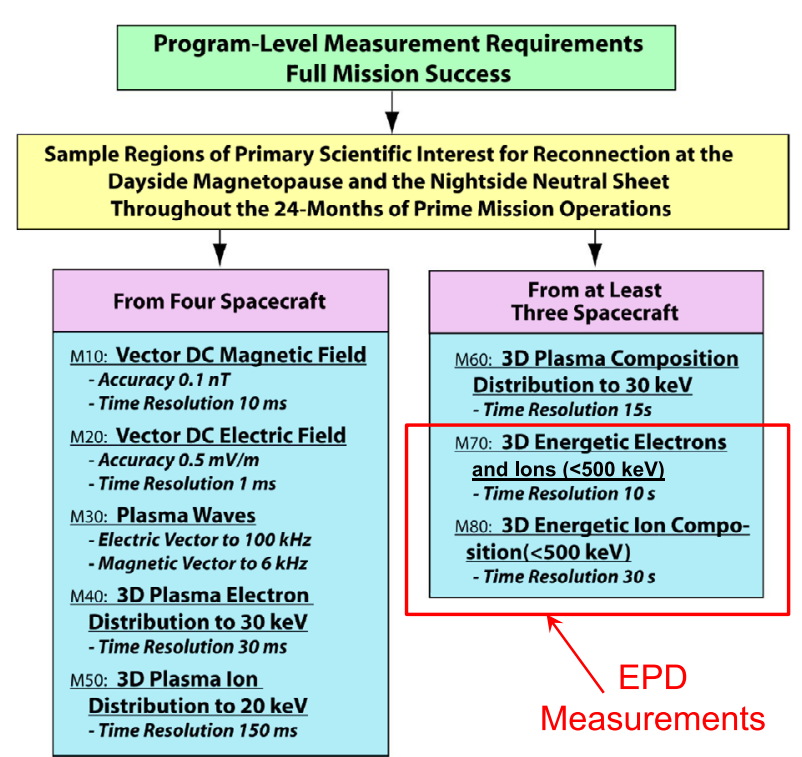


Table 2 Flowdown from EPD science objectives to measurement requirements

\begin{tabular}{|c|c|c|}
\hline $\begin{array}{c}\text { Science } \\
\text { Objectives }\end{array}$ & $\begin{array}{l}\text { 1) } \\
\text { 2) } \\
\text { 4) }\end{array}$ & $\begin{array}{l}\text { Remotely Sense and measure the positions and speeds of boundaries and other structures } \\
\text { Sense magnetic topology with energetic electrons } \\
\text { Remotely sense reconnection acceleration sites } \\
\text { Determine causes of energization and phase space density modifications of energetic } \\
\text { electrons and ions }\end{array}$ \\
\hline $\begin{array}{l}\text { Quantitative } \\
\text { Drivers }\end{array}$ & $\begin{array}{l}\text { Ele } \\
1 . \\
2 . \\
3 . \\
4 . \\
5 . \\
6 .\end{array}$ & $\begin{array}{l}\text { tron and lon Intensities versus energy, angle, and composition such that: } \\
\text { Energies >> electric potentials to sense the magnetic geometry free of electrostatics } \\
\text { Electron all-sky views in time for } 100 \mathrm{~km} / \mathrm{s} \text { motion over } 1 \text { electron gyro-radii } \\
\text { Total ion all-sky views in time for } 100 \mathrm{~km} / \mathrm{s} \text { motion over } 0.5 \text { proton gyro-radii. } \\
\text { Multiple ion / electron energies and resolution for energy/time dispersion analysis. } \\
\text { lon composition to quantify distances, probe scale sizes and acceleration processes. } \\
\text { Sufficient sensitivity for } 25 \mathrm{RE} \text { ions and electrons at the needed time scales }\end{array}$ \\
\hline $\begin{array}{l}\text { Measurement } \\
\text { Requirements }\end{array}$ & $\begin{array}{l}1 . \\
2 . \\
3 . \\
4 . \\
4 . \\
5 . \\
6 . \\
7 . \\
7 .\end{array}$ & $\begin{array}{l}\text { Electron energies: } 25 \mathrm{keV}-500 \mathrm{keV} \text { for remote sensing. lon energies: } 20 \mathrm{keV}-1000 \mathrm{keV} \text { for } \\
\text { probing boundaries and particle acceleration } \\
\text { Electron sampling time for coarse and full electron distributions: } 0.5 \mathrm{~s} \text { and } 10 \mathrm{~s} \text {. } \\
\text { Total-ion / Composition-ion complete angle sampling time: } 10 \mathrm{~s} / 30 \mathrm{~s} \text {. } \\
\text { lon and electron energy resolution: } \Delta \mathrm{E} / \mathrm{E}<50 \% \\
\text { Electron angular resolutions: }<60 \text { degrees for remote sensing, }<30 \text { degrees for probing } \\
\text { particle acceleration } \\
\text { lon angular resolution: }<45 \text { degrees for remote sensing and probing particle acceleration } \\
\text { lon composition requirements: distinguish } \mathrm{H} \text { and } O \\
\text { Electron / ion geometric factors }\left(\mathrm{cm}^{2} \mathrm{sr} \text { per pixel): } \mathrm{G}_{\mathrm{e}}>0.02 \mathrm{~cm}^{2} \mathrm{sr} \text { per pixel; } \mathrm{G}_{1}>0.003 \text {. }\right.\end{array}$ \\
\hline
\end{tabular}

Table 3 Allocation of EPD measurement requirements between EIS and FEEPS

\begin{tabular}{|c|c|c|c|}
\hline Level-1 Drivers & FEEPS $(1-7)$ & Required & Goal (Capability) \\
\hline \multirow[t]{2}{*}{$\begin{array}{l}\text { STP-MMS-M70 } \\
\text { STP-MMS-190 }\end{array}$} & 1. Energy range: & $\begin{array}{l}\text { Elec.:25-500 keV } \\
\text { lons:45-500 keV }\end{array}$ & $\begin{array}{l}\text { Elec.:20-1000 keV } \\
\text { lons:40-1000 keV }\end{array}$ \\
\hline & 2. Energy resolution: & $\begin{array}{c}\text { Elec.: } 50 \% \\
\text { lons: } 50 \% \text { at }>100 \mathrm{keV}\end{array}$ & $\begin{array}{c}\text { Elec.: } 35 \% \\
\text { lons: } 35 \% \text { at }>100 \mathrm{keV}\end{array}$ \\
\hline STP-MMS-M70 & 3. Time elect. fine/course angle coverage & $10 \mathrm{sec} / 0.5 \mathrm{sec}$ & $4 \mathrm{sec} / 0.33 \mathrm{sec}$ \\
\hline \multirow[t]{5}{*}{ STP-MMS-190 } & 4. Angle Coverage & $3.2 \pi \mathrm{sr}$ & $4 \pi \mathrm{sr}$ \\
\hline & 5. Electron (deconvolved) / lon Angle Res. & $30 \mathrm{deg} / 60 \mathrm{deg}$ & $25 \mathrm{deg} / 60 \mathrm{deg}$ \\
\hline & 6. Sensitivity electron (Pixel / Total) $\mathrm{cm}^{\wedge} 2 . \mathrm{sr}$ & $0.02 / 0.36$ & $0.04 / 0.72$ \\
\hline & 7. Sensitivity lons (Pixel/ Total) $\mathrm{cm}^{\wedge} 2 . s r$ : & $0.003 / 0.02$ & $0.01 / 0.06$ \\
\hline & EIS (1-7) & Required & Goal (Capability) \\
\hline \multirow{2}{*}{$\begin{array}{l}\text { STP-MMS-M80 } \\
\text { STP-MMS-190 }\end{array}$} & 1. Energy (protons): & $20-500 \mathrm{keV}$ & $10-1000 \mathrm{keV}$ \\
\hline & 2. Energy resolution: & $50 \%$ at $<100 \mathrm{keV}$ & $35 \%$ at $<100 \mathrm{keV}$ \\
\hline $\begin{array}{l}\text { STP-MMS-M80 } \\
\text { STP-MMS-190 }\end{array}$ & 3. Time: all sky coverage. & $30 \mathrm{sec}$ & $20 \mathrm{sec}$ \\
\hline \multirow[t]{2}{*}{ STP-MMS-190 } & 4. Angle coverage: & $3.2 \pi \mathrm{sr}$. & $3.2 \pi \mathrm{sr}$. \\
\hline & 5. Angular resolution & $<45$ deg. & $<30$ deg. \\
\hline \multirow[t]{3}{*}{ STP-MMS-M80 } & 6. Composition & $\mathrm{H}$ and $\mathrm{O}$ & $\mathrm{H}, \mathrm{He}(>70 \mathrm{keV})$, and $\mathrm{O}$ \\
\hline & 7. Sensitivity lons (Pixel/Total) $\mathrm{cm}^{\wedge} 2 . \mathrm{sr}$ & $0.003 / 0.02$ & $0.005 / 0.03$ \\
\hline & EPD-1 (EIS + FEEPS) & Required & Goal (Capability) \\
\hline $\begin{array}{l}\text { STP-MMS-M70 } \\
\text { STP-MMS-I90 }\end{array}$ & 1. Time: Total ion angle coverage & $10 \mathrm{sec}$ & $7 \mathrm{sec}$ \\
\hline
\end{tabular}

Science Objectives, stated in Sect. 1.2, to the EPD measurement requirements. Finally, Table 3 shows how those measurement requirements are allocated between the FEEPS and the EIS sensors. This table also shows with the left column the connection that the detailed measurement requirements have to various Level-1 requirements. Here, the " $\mathrm{M}$ " require- 


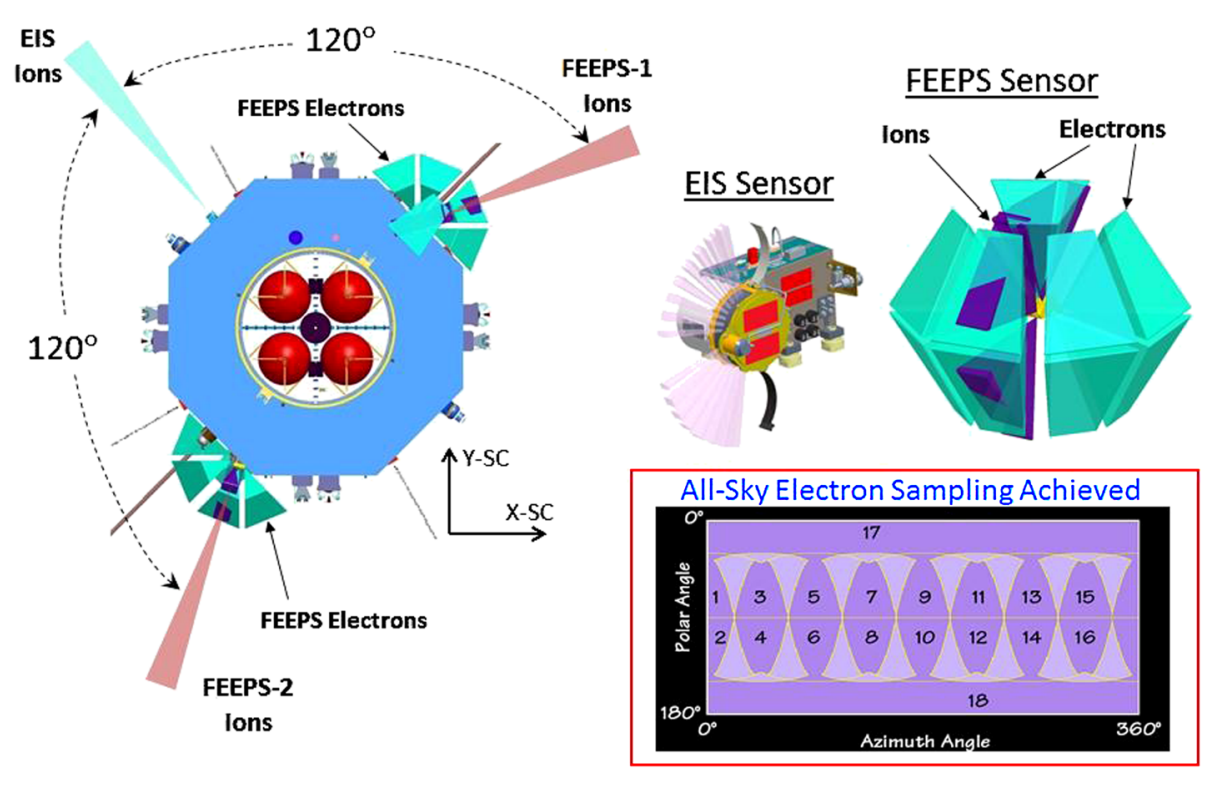

Fig. 8 Several illustrations of the all-sky angle sampling capabilities of the EPD suite of instruments. For the FEEPS sensor viewing illustrated in the upper right, the overlapping electron and ion fields-of-view (FOVs) somewhat obscure the fact that the FEEPS ions on each of the two sensors have 3 elements that are configured to form a fan-like shape

ments (Measurement) are the same as those shown in Fig. 7. The Level-1 "I" requirements (Instrument) add additional quantitative details to the Level-1 "M" requirements.

\section{EPD and EIS Challenges}

The challenges that the EPD team had in meeting the requirements in Sect. 2 revolve around viewing, signal contamination, sensitivity, and solar contamination.

\subsection{Viewing Challenges}

To meet the viewing requirements using spacecraft that spin as slowly as 3 rotations per minute (20 second period; necessary for rotational stability with the long axial booms) it is necessary to view in many directions simultaneously (Fig. 8). To meet the Measurement Requirement \#2 in Table 2 (for timing), it was necessary that the electron sensors view nearly $4 \pi$ steradians at every instant of time. Hence, 2 electron instruments per spacecraft (FEEPS) are required, each with multiple electron views ( 9 , for a total of 18 views per spacecraft). To meet the Measurement Requirement \#5 in Table 2 (for angular resolution), it might have taken many more views per senor. Our plan in meeting that requirement is to oversample in angle (64 samples per spin for all of the multiple electron sensors) and to deconvolute the rotational response by a factor of 2 under the reasonable assumption that the electron distributions are gyro-tropic; that is symmetric with respect to rotations about the magnetic field.

We must also achieve all sky viewing of the ions, but with a lower required cadence $(<10 \mathrm{~s})$. That cadence requirement is achieved robustly by having 3 viewing fans (two 
constructed from multiple FEEPS ion views and one constructed using EIS) viewing into 3 different spacecraft-longitudes, separated by 120 degrees (Fig. 8). For the nominal spin rate of the spacecraft (3 RPM), the all-sky ion viewing is achieved in $\sim 7 \mathrm{~s}$.

\subsection{Signal Contamination Challenges}

Here we address various signal contaminations of the EPD instrument measurements by out of band electrons and ions. We summarize FEEPS issues that will be addressed more completely in the companion paper by Blake et al. (this issue). We note that while EIS has some signal contamination issues of its own discussed here, our general philosophy, given mass and cost constraints, has been to use the instruments more prone to signal contamination (FEEPS) to view in the greatest number of directions to quickly fill out the needed viewing solid angles, and to use the sensor less prone to signal contamination (EIS), viewing a much more restricted part of the sky, in a way that allows us to diagnose the contamination that might be occurring within FEEPS.

FEEPS Electrons Because of limitations in mass and cost, the use of a multiplicity of sensors requires that each sensor be simple. Because each of the FEEPS sensors is simple they are subject to signal contamination. The FEEPS electron sensors (Eyes) comprise simple shaped entrance slots, followed by a 1.8 micron aluminum foil, followed by a single shaped, $1 \mathrm{~mm}$ thick, Solid State Detector (SSD). The aluminum foil is intended to keep energetic ions out. But, in order to allow electrons with energies $>25 \mathrm{keV}$ to be measured, it is necessary to also let protons in with energies $>250 \mathrm{keV}$, yielding contamination of the election measurements under some conditions (particularly when Earth's magnetosphere is bathed in transient solar proton events). Quantitative details of this contamination are provided in the companion paper that focuses on FEEPS (Blake et al. this issue).

FEEPS Ions The FEEPS ion sensors are similarly simple for the same reasons: multiplicity, mass, and cost. The FEEPS ion sensors comprise simple shaped entrance slots followed by 8-10 micron thick shaped SSD's. The SSD's are exceedingly thin in order to minimize their responses to electrons (electrons tend to penetrate and leave energy that is below the set detection threshold). But, none-the less there will be some electron contamination within the lower energy ion channels. Quantitative details of this signal contamination are provided in the companion paper that focuses on FEEPS (Blake et al. this issue). However, as we describe here, the more complete measurements from EIS help FEEPS determine the levels of any signal contamination.

EIS Ions EIS uses time-of-flight $(\mathrm{TOF})$ and TOF $\times$ Energy $(\mathrm{E})$ coincident measurements to strongly minimize signal contamination by electrons and other random inputs (UV light). A schematic of the EIS sensor is shown in Fig. 9 left. Ions enter from the left, pass through a thin "Collimator Foil" (discussed below), go on to generate secondary electrons within a thin "Start Foil", go on to generate secondary electrons in a "Stop Foil", and then, if they are energetic enough, are detected by one of the SSD's in the back array of SSD's on the right. The secondary electrons from the start and stop foils are electrostatically steered onto the microchannel plate (MCP) which delivers a cloud of electrons to one of the collection anodes on each side. The arrival direction (one of 6) is measured by the determination of which anodes are illuminated. The velocity of the particle is determined by the time difference (TOF) between the start and stop region, and the energy is determined by the deposition in the SSD's. Ion energy and species can be determined by channelization of the TOF $\times$ E parameters. For particles too low in energy to stimulate the SSD's, a TOF $\times$ MCP-Pulse-Height 

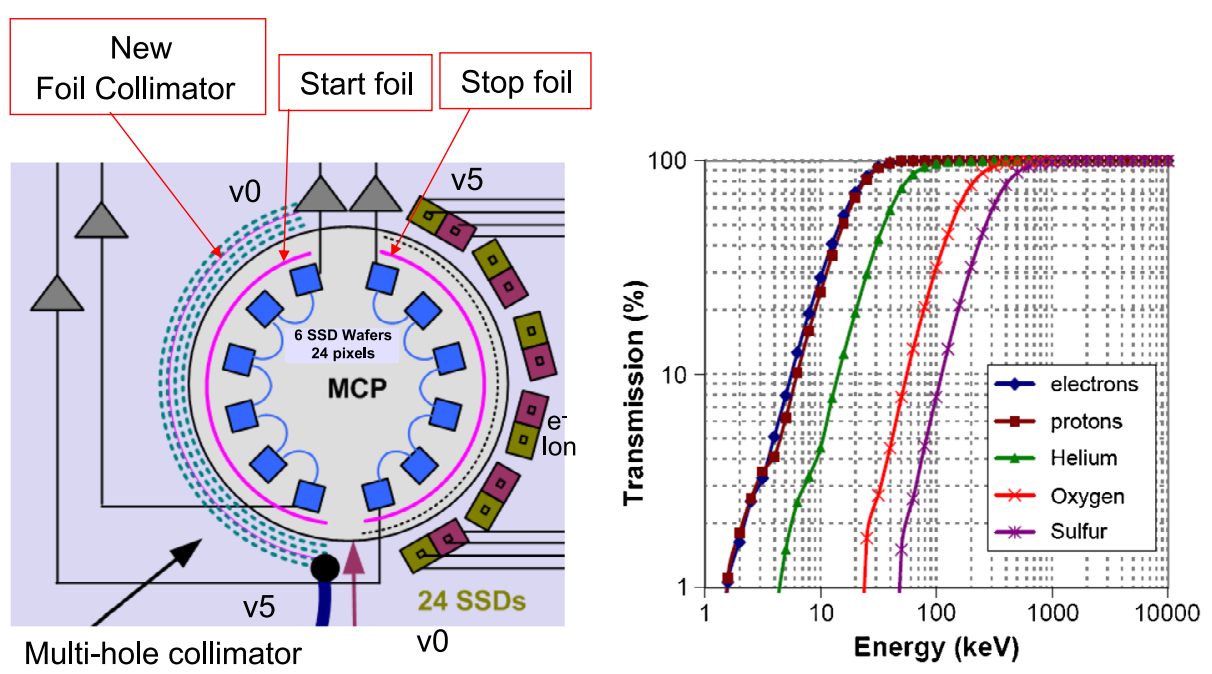

Fig. 9 In order to suppress the contribution of out-of-band low energy electron and ions to the response of the EIS foils, a 3rd foil, the "collimator foil," was added to the collimator of EIS. The right panel shows a GEANT4 estimate of that suppression as a function of energy for various species

$(\mathrm{TOF} \times \mathrm{PH})$ product is generated which determines the particle Energy/nucleon and provides a crude species determination (roughly separating $\mathrm{H}$ and $\mathrm{O}$ ).

The fact that a valid event requires coincident measurements (start, stop, and perhaps SSD) with short time windows means that EIS is relatively immune from the random stimulations that come from electrons and UV light. However, the fluxes of energetic electrons can be intense enough that making EIS relatively immune to energetic electrons was a challenge. When the start foil and the stop foil are driven to very high random rates, there will occur what are called "accidental" events that are interpreted by the sensor as valid events. Total accidental rates are of order $\mathrm{T}_{\mathrm{W}} \times \mathrm{R}_{\mathrm{START}} \times \mathrm{R}_{\mathrm{STOP}}$, where $\mathrm{T}_{\mathrm{W}}$ is a time window (e.g. the maximum time it takes a low energy oxygen ion to traverse the sensor volume, about $160 \mathrm{nsec}$ ), $\mathrm{R}_{\text {START }}$ is the random start rate stimulated by electrons or UV light, and $\mathrm{R}_{\text {STOP }}$ is the random stop rates. For start and stop rates $>10^{5}$ accidental rates may start to compete with real foreground rates.

While it is conventional wisdom that energetic electrons do not interact with foils nearly as readily as do ions, electrons below a couple of keV interact very strongly (Mauk et al. 2013; Fig. 9 of that paper). EIS has some protection from keV-class electrons because they must overcome a $\sim 2.6 \mathrm{kV}$ potential hill before reaching the start foil (the voltage is a part of the secondary electron optics; see later discussion). However, even with this potential barrier, early modeling of the EIS sensor response to electron distributions that will be found in MMS target regions suggested that there would be times when EIS ion measurements would be substantially contaminated by accidentals from electrons. Dealing with that issue is the purpose of the thin aluminum collimator foil positioned within the center of the collimator (Fig. 9, left). This foil causes the majority of very low energy electrons to scatter such that they strike collimator blades (see later discussion) and do not make it into sensor volume. The right panel shows the modeling of the suppression of the lower energy response of the sensor. In order to achieve this suppression for electrons, we had to also accept the suppression for lower energy ions as well, a compromise that we needed to make. 
Fig. 10 Drawing of the internal EIS sensor configuration highlighting the design of the collimator, to be flown for the first time with EIS. The solid state detector array, with 6 elements, is shown at the top. The gap in the response along the vertical symmetry line is there to prevent sun-light from directly impinging on the EIS start foil

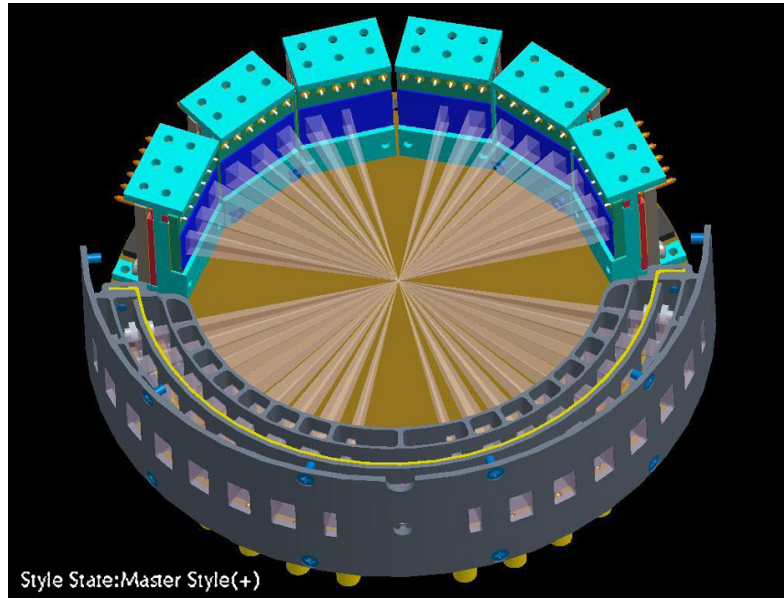

EIS Electrons EIS is not required to measure electrons. However, because the parent sensor to EIS, the JEDI instrument on Juno (Mauk et al. 2013) had an electron capability, and because it was judged that this capability would be useful to sorting out ion contamination of electrons, we decided to keep this capability. Figure 9 (left) shows that the SSD array contains both electron and ion SSD pixels. The electron SSD pixels are different in that they have a 2 micron aluminum flashing on them that keeps $<250 \mathrm{keV}$ protons from reaching the SSD. In other words, EIS measures electrons in exactly the same way that FEEPS measures electrons (the MCP and foils do not play a role in the measurement of the electrons). The difference for EIS is that the electron fields-of-view are very close to the ion fields-of-view and have the same shapes. The fields-of-view alternate between electrons and ions. In this way, EIS is much better configured to determine whether or not the electron sensors are contaminated by energetic ions since the ion sensors and electron sensors are simultaneously sampling nearly the same regions of space. A deficiency in the EIS sensor's ability to measure electrons (as contrasted with JEDI which is constructed of much denser materials, and in contrast to FEEPS) is that the amount of shielding against penetrating very high energy electrons is less than ideal for an energetic electron sensor (Sect. A.5).

\subsection{Sensitivity Challenges}

FEEPS Electrons For FEEPS electrons, our objective was to be able to detect very fast changes as the spacecraft traversed the very small spatial regions that comprise the reconnection sites. Our original goal was to obtain a total energetic electron geometric factor $\left(\epsilon \mathrm{G}\right.$, where $\epsilon$ is efficiency and $\mathrm{G}$ is $\mathrm{cm}^{2} \mathrm{sr}$ ) for each spacecraft of order 1.0. As indicated in Table 3, we achieved a spacecraft total with all sky viewing of about 0.7 . What that value achieves for us in sensitivity to prevailing electron intensities is addressed in the companion paper by Blake et al. (this issue).

EIS Ions Cost drove us to stay very close to an existing design with EIS. That design is the Juno JEDI sensor launched in August of 2012 (Mauk et al. 2013). The total geometric factor of the JEDI sensor is about $0.01 \mathrm{~cm}^{2} \mathrm{sr}$. That value was judged to be too small to meet the minimum needs of EIS. Our goal was to maximize the geometric factor of the existing sensor design while maintaining other required characteristics, for example angular resolution. Aspects of the EIS design are illustrated in Fig. 10. Rather than the multi-holed 

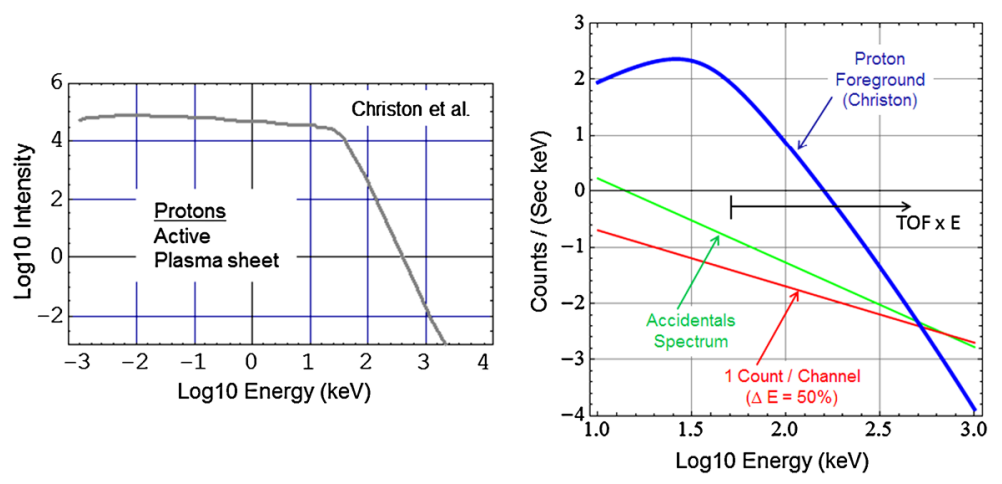

Fig. 11 Characteristic ion spectrum sampled in an active Earth plasma sheet, and the EIS instrument response to that spectrum (Spectrum fitted from Christon et al. 1991). The measurement limitations are also shown on the right panel: counting statistics and the limitations imposed by so-called accidentals caused by electrons and UV light interacting with the start and stop foils. The "Accidentals" limit does not contribute to the noise when $\mathrm{TOF} \times \mathrm{E}$ is used above $50 \mathrm{keV}$ for $\mathrm{H}+$ and above $150 \mathrm{keV}$ for $\mathrm{O}+$

collimator of JEDI (about 90 circular holes arranges in 3, hexagonally positioned rows of 30 holes), the EIS collimator comprises 16 slots within existing 5 cylindrically concentric blades, with slot sizes that converge to zero size when they are extrapolated to the center of the sensor (Fig. 10). The resulting geometric factor is greater by more than a factor of 3 as compared to the JEDI design. That value is judged to be adequate for MMS but not generous. What we have given up is angular resolution from 18 degrees achieve by JEDI with the help of rotation, to the EIS goal of 30 degrees. In order to prevent substantial side lobes to the angular response, it was also necessary to incorporate radial fins between the outer two collimator blades and between the inner two collimator blades (Fig. 10)

An active plasma sheet ion spectrum (Christon et al. 1991) that EIS needs to be able to characterize is shown in Fig. 11 (left). The EIS response to that spectrum (in terms of Counts/(sec keV)), including all energy loss and efficiency terms, is shown in Fig. 11 (right), along with comparisons to the measurement limitations. The "accidentals" spectrum is based on an active electron spectrum also published by Christon et al. (1991), but because of additional coincidence contraints, that contribution to the noise is irrelevant for protons energies $>50 \mathrm{keV}$ when TOF $\times \mathrm{E}$ will be used ( $>150 \mathrm{keV}$ for oxygen).

\subsection{Solar Contamination Challenge}

Because the spin axis of MMS is roughly (but not exactly) perpendicular to the sunspacecraft line, and because EIS aspires to yield all-sky measures of the ion populations, there was the potential that EIS would directly view the sun once per spin, contaminating the measurements for a substantial swath of angular sampling. Thus, the collimator also had to be designed so that the sun could not have direct access to the sensor volume. Figure 10 shows that the collimator in the region closest to the symmetry axis of the field of view has no entrance slots in order to prevent sunlight from striking the start foil directly. The sun will gain access to the start foil if the elevation angle of the sun with respect the symmetry axis of EIS is more than about 7 degrees (note that the $+/-0.25$ degree size of the sun must be incorporated into calculations). The project accepted a requirement that the $\mathrm{Z}$-axis will be kept at an angle with respect to the sun that keeps the sun out of the EIS field of view. There will be scattering within the collimator, and there will be some level of solar 


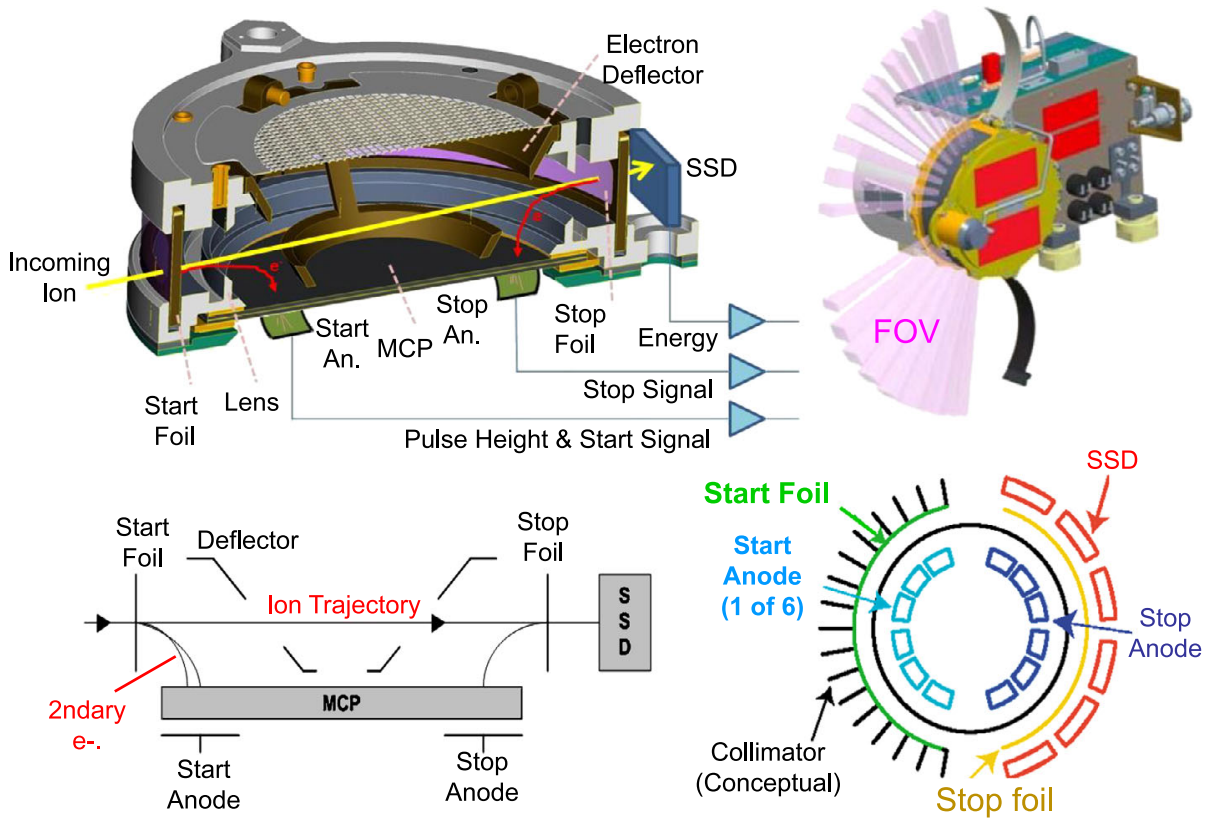

Fig. 12 Exhibits showing how each EIS sensor operates

contamination when the EIS field-of-view is pointing in the general direction of the sun, but the configuration described here will minimize that contamination.

FEEPS had a similar solar contamination problem for its ion measurements (not electrons) that are addressed in Blake et al. (this issue).

\section{The EIS Instrument}

Here we describe in some detail the design, hardware and inner workings of the EIS instrument. Each instrument comprises two subsystems, the sensor head and the main electronics (Fig. 12, upper right). Each sensor head incorporates electron and ion sensors (e.g. Fig. 9 and later discussions), plus detector preamplifiers. The sensor head and main electronics are mechanically integrated together and mounted as a single unit to the spacecraft.

Note that to keep the technical descriptions simple and straight forward, we do not provide many of the technical specifications for the EIS instrument in this section (mass, power, sizes, materials, densities, thicknesses, gaps, voltages, etc.). Those specifications are provided in the Appendix.

\subsection{Principles of Operation}

EIS measures ion energy, directional, and compositional distributions using Time-of-Flight by Energy $(\mathrm{TOF} \times \mathrm{E})$ and Time-of-Flight by MCP-Pulse-Height $(\mathrm{TOF} \times \mathrm{PH})$ techniques. EIS measures electron energy and directional distributions using collimated solid-statedetector (SSD) energy measurements (these electron SSD's, as opposed to the ion SSD's, have 2 microns of aluminum flashing deposited on them to keep out protons with energies 
less than about $250 \mathrm{keV}$ ). EIS combines multidirectional viewing into individual compact sensor heads (Fig. 12). The sensor heads include time-of-flight (TOF) sections about $6 \mathrm{~cm}$ across feeding a solid-state silicon detector (SSD) array. Secondary electrons, generated by ions passing through the entry and exit foils (Fig. 12 left), are detected by the microchannel plate (MCP) stimulated timing anodes and their associated preamps to measure ion TOF. Event energy (E) and TOF measurements are combined to derive ion mass and to identify particle species.

The EIS acceptance angle is fan-like and measures $160^{\circ}$ by $12^{\circ}$ with six $\sim 26.7^{\circ}$ look directions (this pattern is quantitatively disrupted for the two central pixels due to the solar blockage-Fig. 10). Particle direction is determined by the particular look direction in which it is detected (six different view directions for each species, labeled v0, v1, v2, v3, v4 and v5). That directionality is determined by the active SSD in the case of electrons, and by the determination of the entrance position on the MCP-stimulated time-delay anode nearest to the start foil in the case of ions (time delay along a chain of 12 "start" anode pads connected by inductors is used to determine entrance position). Ions that pass through the sensor encounter three separate thin foils mounted on $\sim 90 \%$ transmission grids. The first one, the "collimator foil," mounted within the collimator, is a $350 \AA$ aluminum foil. The next foil, the "start" foil, is a $50 \AA$ carbon/350 A polyimide/50 A carbon foil. These 2 foils reduce the UV (e.g. primarily Lyman alpha) photon background. The exit apertures are covered by

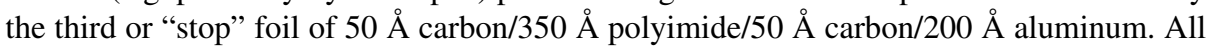
foils are mounted on high-transmittance $(90 \%)$ metal grids supported on a metal frames.

Electron Sensors Before an electron passes through the TOF head, it is first decelerated by a $2.6 \mathrm{kV}$ potential (part of the TOF optics for measuring ions); it is later reaccelerated by $2.6 \mathrm{kV}$ after exiting the stop foil prior to reaching the SSD detectors. Energetic electrons from $25 \mathrm{keV}$ to $1000 \mathrm{keV}$ are measured by the electron SSD detectors. The electron detectors are covered with $2 \mu \mathrm{m}$ aluminum metal flashing to keep out protons and ion particles with energies less than about $250 \mathrm{keV}$. No TOF criterion is applied to the electron measurements. The sensitivity to particles can be adjusted by a factor of 20 by selecting large or small SSD pixels (discussed below).

Ion Sensors As described above, an ion encounters 3 foils on its way to the SSD (Fig. 12 left). Secondary electrons from the start and stop foils are electrostatically separated from the primary particle path and diverted on the microchannel plate (MCP), providing start and stop signals for TOF measurements. The segmented MCP anodes, with two start and two stop anodes for each of the six angular segments, determine the direction of travel. A 500-volt accelerating potential between the foil and the MCP surface, combined with the cone-like electrostatic mirror (labeled "electron deflector" in the upper left of Fig. 12), controls the electrostatic steering of secondary electrons. The dispersion in secondary electron transit time is less than $1 \mathrm{nsec}$. As an aside we should note that after penetrating any foil, the ion may emerge as an ion or as a neutral. If it emerges as an ion from the collimator foil it is subject to the acceleration and/or deceleration potentials $(2.6 \mathrm{kV})$ associated with the secondary electron optics

Ion energy measurements using the ion detectors are combined with coincident TOF measurements to derive particle mass and identify particle species (the TOF $\times \mathrm{E}$ method). With the TOF $\times$ E method the incoming particles are measured from $50 \mathrm{keV}$ to above $1 \mathrm{MeV}$; they are discriminated in the energy system above $50 \mathrm{keV}$ for protons and above about $150 \mathrm{keV}$ for heavy ions (such as the CNO group). An example of a TOF $\times \mathrm{E}$ matrix and how it separates different mass species is shown in the left panel of Fig. 13 from the New 

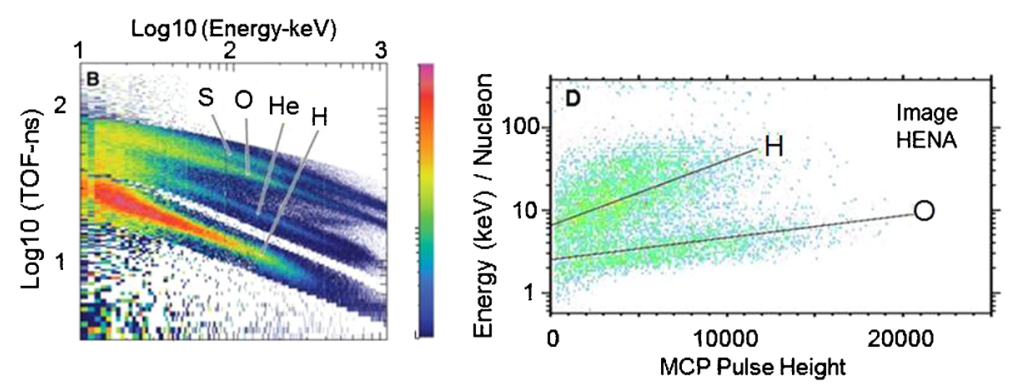

Fig. 13 (left) Measurements made by an EIS predecessor instrument, PEPSSI, during the New Horizons encounter with Jupiter. (right) TOF $\times$ Pulse-Height measurements made at Earth from the IMAGE HENA instrument

Horizons PEPSSI instrument at Jupiter (McNutt et al. 2008). Lower-energy ion fluxes are measured using TOF-only measurements (the TOF $\times$ Pulse Height method); detection of MCP pulse height provides a coarse indication of low-energy particle mass. An example of how a TOF $\times$ PH spectrum crudely separates different ion mass species at Earth, from the IMAGE HENA instrument, is shown in Fig. 13 (right; Mitchell et al. 2003). Sensitivity to higher energy ions (those with energies above the SSD channel thresholds) can be adjusted by selecting large or small SSD pixels. However, this capability is a hold-over from the Juno JEDI instrument. It is unlikely that the small pixels will be needed in the primary MMS target regions.

\subsection{EIS Heritage}

The Johns Hopkins APL has generated and flown numerous TOF $\times \mathrm{E}$ instruments, generally including SSD-based sensors, on numerous spacecraft. The list includes the Earthorbiting AMPTE CCE MEPA instrument (McEntire et al. 1985) and Geotail EPIC instrument (Williams et al. 1994), the Jupiter-orbiting Galileo EPD instrument (Williams et al. 1992), and the New Horizons PEPSSI instrument now on its way to Pluto (McNutt et al. 2008). Instruments that have used the TOF $\times$ PH method include the Earth-orbiting IMAGE HENA instrument (Mitchell et al. 2003) and the Saturn-orbiting Cassini MIMI INCA instrument (Krimigis et al. 2004). The EIS design is heavily based on the JUNO JEDI instrument design (Mauk et al. 2013) which draws its heritage from the New Horizons PEPSSI instrument. The RBSPICE instrument on the Van Allen Probes mission (Mitchell et al. 2013) was also based closely on JEDI.

\subsection{EIS Block Diagram and Details of the Electronic Design}

The electronics box contains all the electronics to run the instrument other than the energy and timing preamps which are located in the sensor head. The box comprises three $10 \times$ $15 \mathrm{~cm}$ boards mounted into $2.5 \mathrm{~mm}$ thick metal frames. The boards stack one on top of the other, with a stacking connector providing electrical interconnects between the boards.

The EIS Block Diagram is shown in Fig. 14. On the left, the sensor generates analog representations of particle timing signals that go into the determination of Time-of-Flight (TOF), and energy E from the SSD's. Each SSD has both electron and ion pixels. There is only one analog electronics processing chain per SSD. Consequently, to collect both electrons and ions, the hardware is time-multiplexed between the electron and ion detectors. The 


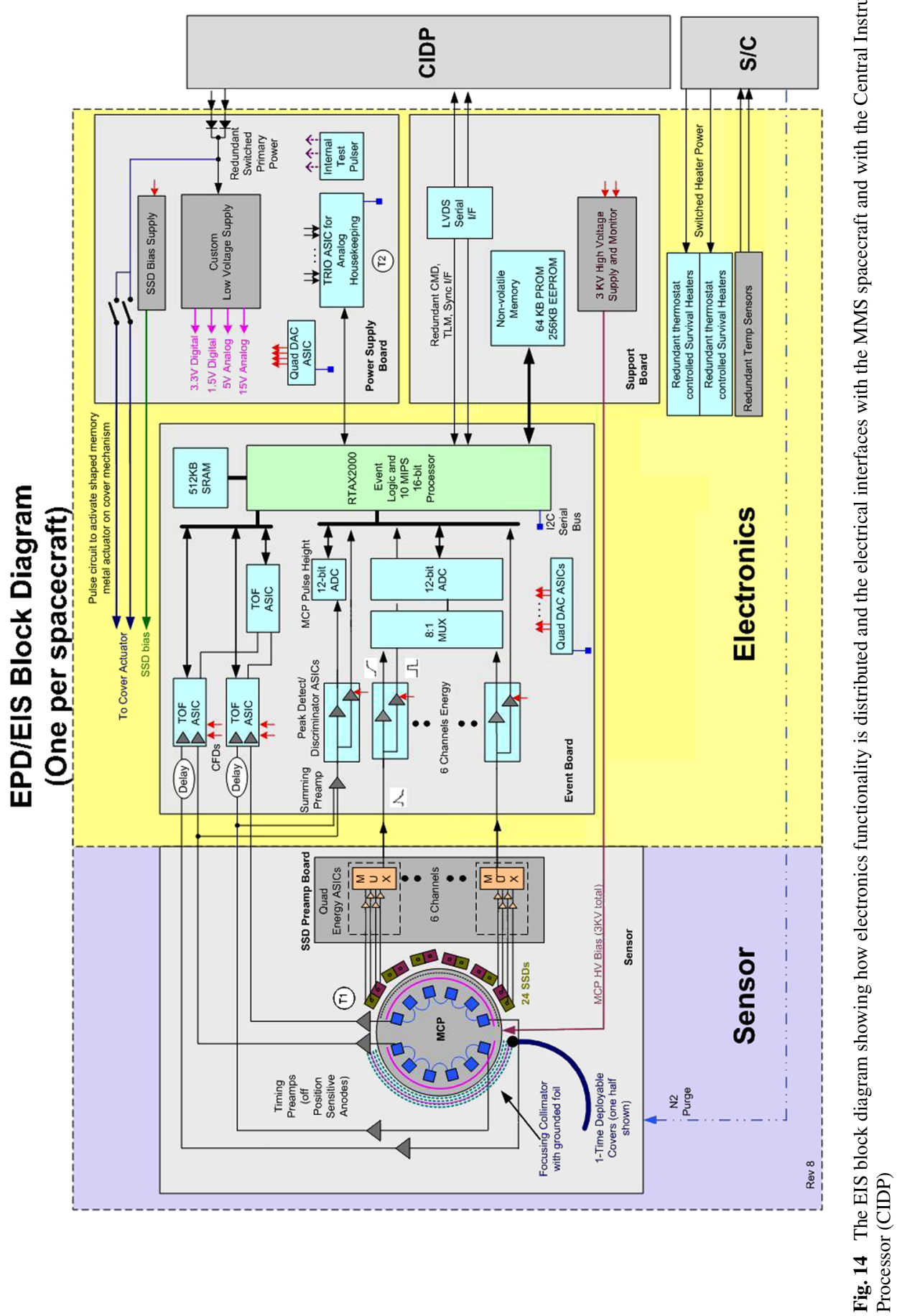


Table 4 Particle event data

\begin{tabular}{lrl}
\hline Name & Bits & Description \\
\hline TOF1 & 10 & TOF chip 1, Stop0-Start0 \\
TOF2 & 10 & TOF chip 2, Stop5-Start5 \\
TOF3 & 10 & TOF chip 3, Start5-Start0 \\
TOF Flags & 3 & TOF chip validity flags (VE3, VE2, VE1) \\
TOF & 10 & Corrected TOF, average of TOF1 and TOF2 \\
Start Chan & 3 & Calculated start channel, 0-5 (6, 7 = invalid) \\
Stop Chan & 3 & Calculated stop channel, 0-5 (6, 7 = invalid) \\
MCP PH & 12 & MCP pulse height \\
MCP PH Flag & 1 & MCP PH flag $(0=$ no pulse, $1=$ pulse $)$ \\
SSD Energy & 12 & SSD energy \\
SSD Coin Flags & 2 & SSD with start; SSD with stop \\
SSD Flags & 6 & SSD flags $(0=$ no pulse, $1=$ pulse) \\
SSD Chan & 3 & SSD channel, 0-5 (6 = no energy) \\
SSD/MCP PW & 9 & SSD (or MCP) pulse width \\
\hline
\end{tabular}

hardware is in fact time-multiplexed between three possible modes: electron energy, ion energy (with no TOF constraint), and ion species. An event trigger selects what combination of TOF and SSD pulses defines an event. With energy trigger, an SSD energy (E) pulse defines an event. With TOF trigger, a TOF pulse, with or without an E pulse defines an event. For EIS, it will be typical to cycle through 2 different species modes ( $3 / 4$ of the time on ion species and $1 / 4$ of the time on electrons) every $\sim 0.67$ seconds (exactly $1 / 32$ of a spin). The EIS hardware passes valid particle event data to the software for further analysis using a First-In First-Out (FIFO) device.

Each ion species event consists of several parameters, which are shown in Table 4. For energy events, only SSD data are valid. For events that trigger the Time-of-Flight system, TOF1, TOF2, and TOF3 are the raw values produced by the three TOF chips. TOF chips are used for determining both the times of flights of the particle through the system and the arrival directions of the particles using time-delay anodes on the start and the stop portions of the anodes. TOF1 and TOF2 provide redundant measurements of the particle's time-offlight. TOF1 measures the time between the Start 0 and Stop0 pulses $(0$ being one end of the time-delay anodes); TOF2 measures the time between the Start5 and Stop5 pulses ( 5 being the other end of the time-delay anodes). TOF3 measures the time between the Start0 and Start5 pulses; this provides the particle's position on the start anode. The "TOF" parameter in Table 4 provides the corrected TOF value, the average of TOF1 and TOF2. The start position is measured by TOF3. The stop position, i.e. the time between Stop0 and Stop5, is calculated in the FPGA as TOF2 + TOF3 - TOF1; the result is not reported in the event data. The start position and the stop position are used to calculate a start direction and a stop direction, respectively. Note that various levels of agreement between the start, stop positions, and SSD can be enforced by EIS firmware and software to restrict the ranges of ion path lengths for any one view direction; for example one may set the parameter " $n$ ", in the equation "Stop_Position = Start_Position \pm n", where $\mathrm{n}$ can be 0,1 , or 2 .

Event Board The event board digitizes the TOF, the MCP Pulse Height (PH), and the SSD energy E; and reads the events into a Field Programmable Gate Array (FPGA). The FPGA 
contains event processing logic and a processor. Some events are passed to software running on the processor for further analysis and science processing. The Event board directly processes the sensor SSD and anode preamp output signals, and contains all the necessary analog and digital circuitry to process and store event information on an event-by-event basis. The energy signals from the six SSD preamplifiers and the MCP anode pulse height are processed in parallel peak-detect/discriminator circuit and multiplexed into a single 12 bit ADC. The MCP anode signals are processed via constant-fraction discriminators (CFDs) and time-to-digital (TDC) circuitry; these measured time differences are converted into event look direction and particle velocity in the FPGA. FPGA-based event logic also determines which signals comprise valid ion and electron events and coordinates all event hardware processing timing. An APL-developed soft-core processor, the Scalable Configurable Instrument Processor (SCIP) is also embedded in the FPGA to provide all command, control, telemetry, and data processing functions of the instrument (Hayes 2005). SRAM memory storage is provided on the board to support this processor. EEPROM and boot PROM support is provided on the Support Board. The Event board plugs into the Support and Power boards.

Support Board The Support board provides a variety of support functions for the instrument. It also contains EEPROM and boot PROM accessible to the FPGA on the Event Board. The command and telemetry interface to the spacecraft is provided here. The board includes the high voltage power supply, which generates the necessary high voltage outputs for the sensor MCP and electron optics; maximum voltage is $3300 \mathrm{~V}$. The Support board plugs into the Event and Power boards.

Power Board The Power board contains both the low and SSD bias voltage power supplies. The low voltage portion takes spacecraft primary power on a single 9 pin connector and generates $1.5 \mathrm{~V}$ (for FPGA core), $3.3 \mathrm{~V}$ (primarily for digital interface logic, memories, and TDCs), and $5 \mathrm{~V}$ (primarily for analog functions). A $15 \mathrm{~V}$ output powers the high voltage electronics on the support board. The board also switches power to the sensor cover actuator mechanism and generates and filters $100 \mathrm{~V}$ bias for the SSD detectors. The board plugs into both the Event and Support boards.

ASICS EIS utilizes five different APL-developed rad-hard ASICs in its electronics (Paschalidis et al. 2002; Paschalidis 2006). It has three APL TOF-C ASICs to measure the "Start" (entrance) and "Stop" (exit) positions on the sensor timing anodes and the timeof-flight for ions traveling between the Start and Stop foils. The TOF ASICs, which also incorporate very fast constant fraction discriminator front ends, are configured to measure times between 0 and $32 \mathrm{nsec}$ with $50 \mathrm{psec}$ resolution (anode positions) and between 0 and $160 \mathrm{nsec}$ with $200 \mathrm{psec}$ resolution (time-of-flight). Each of EIS's six look direction utilizes a Quad Energy Chip (preamp/shaper) ASIC followed by a peak detector/discriminator ASIC to process the 4-pixel solid state detector (SSD) arrays. EIS's control circuitry utilizes a 16channel TRIO ASIC to multiplex and perform 10-bit analog to digital conversion of analog status information, and a number of Quad 8-bit DACs to set thresholds and control high voltage and SSD bias levels; the TOF ASICs communicate with the instrument FPGA via a parallel interface, while the Quad DAC and TRIO use serial I2C interfaces. The ASICs each require between 5 and $25 \mathrm{~mW}$, and all inherently meet performance requirements beyond 100 krad radiation dose. 
Table 5 EIS hardware modes

\begin{tabular}{|c|c|c|c|}
\hline Resource & Electron energy & Ion energy & Ion species \\
\hline Event Trigger & \multicolumn{2}{|l|}{ Set energy trigger } & Set TOF trigger \\
\hline TOF CFD Thresholds & \multicolumn{3}{|l|}{ Set TOF CFD thresholds } \\
\hline $\begin{array}{l}\text { TOF Pulse Height } \\
\text { Threshold }\end{array}$ & \multicolumn{2}{|c|}{ N/A: set TOF pulse height threshold to max } & $\begin{array}{l}\text { Set TOF pulse height } \\
\text { threshold }\end{array}$ \\
\hline $\begin{array}{l}\text { Electron vs. Ion } \\
\text { Detector }\end{array}$ & Set electron source & Set ion source & $\begin{array}{l}\text { Set ion (or electron) } \\
\text { source }\end{array}$ \\
\hline Pixel Size & $\begin{array}{l}\text { Set pixel size selected } \\
\text { for electron }\end{array}$ & $\begin{array}{l}\text { Set pixel size selected } \\
\text { for ion energy }\end{array}$ & $\begin{array}{l}\text { Set pixel size selected } \\
\text { for ion species }\end{array}$ \\
\hline $\begin{array}{l}\text { Energy Channel } \\
\text { Enable/Disable }\end{array}$ & $\begin{array}{l}\text { Set energy mask for } \\
\text { electron and selected } \\
\text { pixel size }\end{array}$ & $\begin{array}{l}\text { Set energy mask for ion } \\
\text { and selected pixel size }\end{array}$ & $\begin{array}{l}\text { Set energy mask for ion } \\
\text { and selected pixel size }\end{array}$ \\
\hline Energy Thresholds & $\begin{array}{l}\text { Set energy discriminator } \\
\text { thresholds for electron } \\
\text { and selected pixel size }\end{array}$ & $\begin{array}{l}\text { Set energy discriminator } \\
\text { thresholds for ion and } \\
\text { selected pixel size }\end{array}$ & $\begin{array}{l}\text { Set energy discriminator } \\
\text { thresholds for ion and } \\
\text { selected pixel size }\end{array}$ \\
\hline Energy Baselines & $\begin{array}{l}\text { Set energy baselines for } \\
\text { electron and selected } \\
\text { pixel size }\end{array}$ & $\begin{array}{l}\text { Set energy baselines for } \\
\text { ion and selected pixel } \\
\text { size }\end{array}$ & $\begin{array}{l}\text { Set energy baselines for } \\
\text { ion and selected pixel } \\
\text { size }\end{array}$ \\
\hline Coincidence & \multicolumn{3}{|c|}{ Set event coincidence window } \\
\hline Multiple Hit Reject & $\begin{array}{l}\text { Enable/disable multiple } \\
\text { hit reject for election }\end{array}$ & \multicolumn{2}{|c|}{ Enable/disable multiple hit reject for ion } \\
\hline Valid Event & N/A & N/A & $\begin{array}{l}\text { Select valid TOF chips } \\
\text { for ion species }\end{array}$ \\
\hline
\end{tabular}

Harnessing The sensor head is electrically connected to the electronics box via coaxial cables and twisted wire interfaces. These lines are fairly short in length (typically less than $10 \mathrm{~cm}$ ), and are covered by a thin EMI shield to extend the faraday cage between the sensor and electronics housings. The instrument is electrically interfaced to the spacecraft and CIDP via dedicated spacecraft-provided power and data cables.

Instrument Settings Table 5 documents the numerous JEDI hardware modes and parameter settings that can be set by command. Several "standard" settings (setting all of the various parameters shown in the table) are generated by running one of several internal instrument "macros" during the EIS turn-on sequence or by external command at other times. As an example, optimum threshold settings have some sensitivity to temperature, and onboard macros are embedded within the instrument for several temperatures over the operational range.

\subsection{EIS Mechanical Configuration}

External Instrument and Mounting The external mechanical configurations of the four EIS instrument configurations are shown in Fig. 15. The schematic shows the instrument with its one-time deploy acoustic doors deployed, whereas the photographs show the doors closed. EIS, like most other MMS instruments, is mounted to the MMS payload deck (Fig. 2). EIS is mounted from the bottom side of the instrument deck (Fig. 16; on the spacecraft interior side of the payload deck), such that the instrument "look direction 5" is towards the mounting deck (and thus the $+Z$ Spacecraft axis; aligned with the $+Y$ EIS axis), and the instrument "look direction 0" is towards the $-\mathrm{Z}$ axis (Fig. 16; aligned with the $-\mathrm{Y}$ EIS axis). The instrument coordinate system is shown on Fig. 16 in red. 

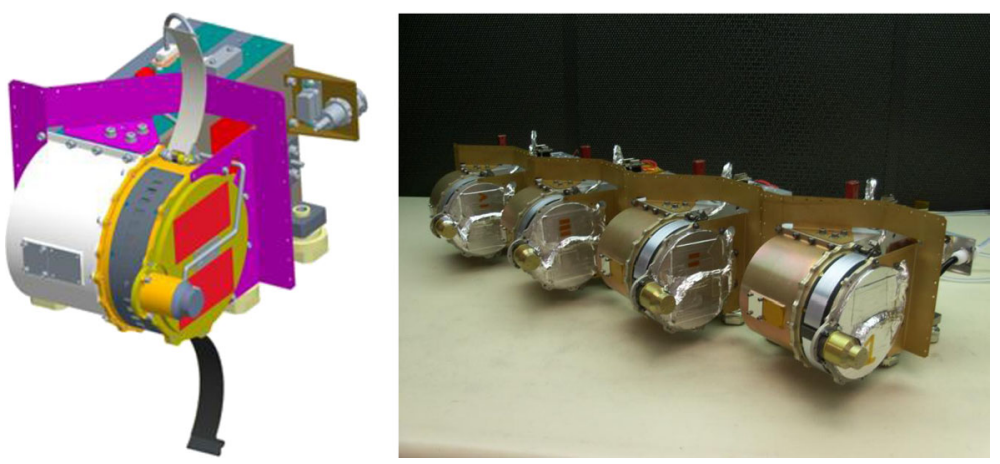

Fig. 15 Schematic and photograph of the external mechanical configurations of the EIS instruments. The schematic shows the 1-time deploy acoustic door in the open position

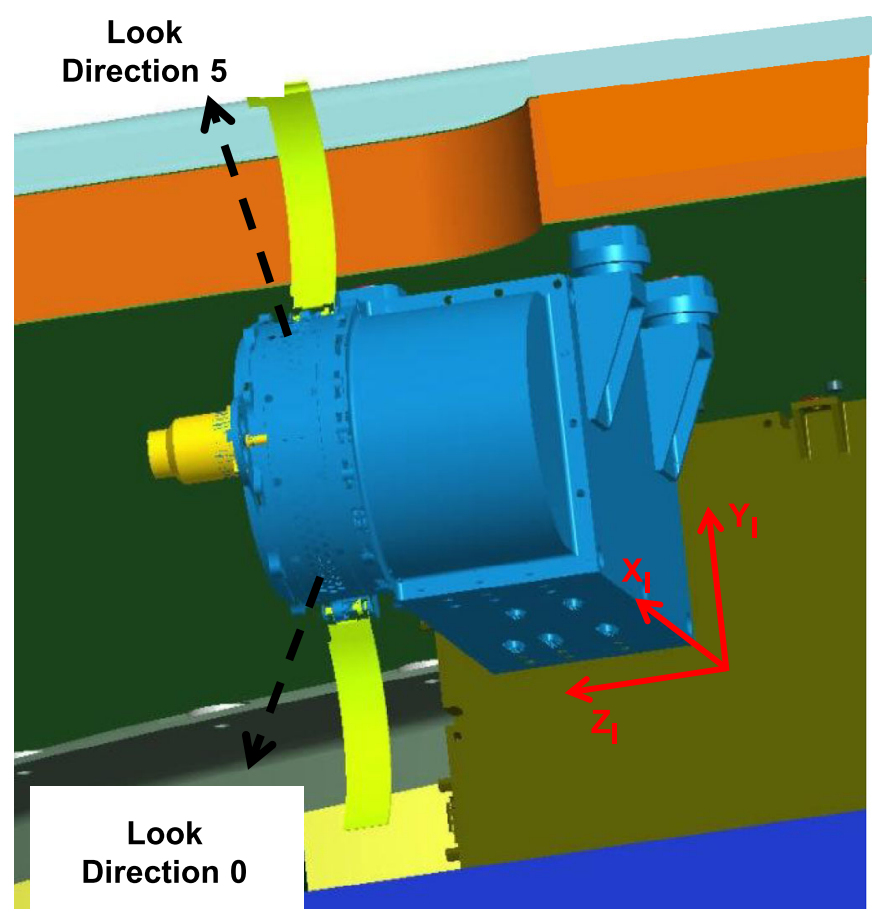

Fig. 16 Details of the mechanical mounting of EIS on the MMS spacecraft with the EIS coordinate system shown in red (the SC coordinate system is shown in Figs. 2 and 8). EIS has 5 distinct view directions (v0, v1, $\mathrm{v} 2, \mathrm{v} 3, \mathrm{v} 4, \mathrm{v} 5)$, and the figure shows the directions of the v0 and v5. V0 tilts towards the SC $-\mathrm{Z}$ axis, and V5 tilts towards the $\mathrm{SC}+\mathrm{Z}$ axis. The $\mathrm{EIS}+\mathrm{Y}$-axis is aligned with the $\mathrm{SC}+\mathrm{Z}$ axis (see the Appendix)

Internal Structure The internal structure of each EIS instrument is shown with the cutaway diagram in Fig. 17. There is a TiNi actuated pin puller that releases the spring-loaded doors. The figure shows some of the internal structure of the sensor, and the positioning of the three main electronics boards. Photographs of selected elements of the sensor are shown in Fig. 18. The upper left hand portion shows the anode board with the energy system mounted 


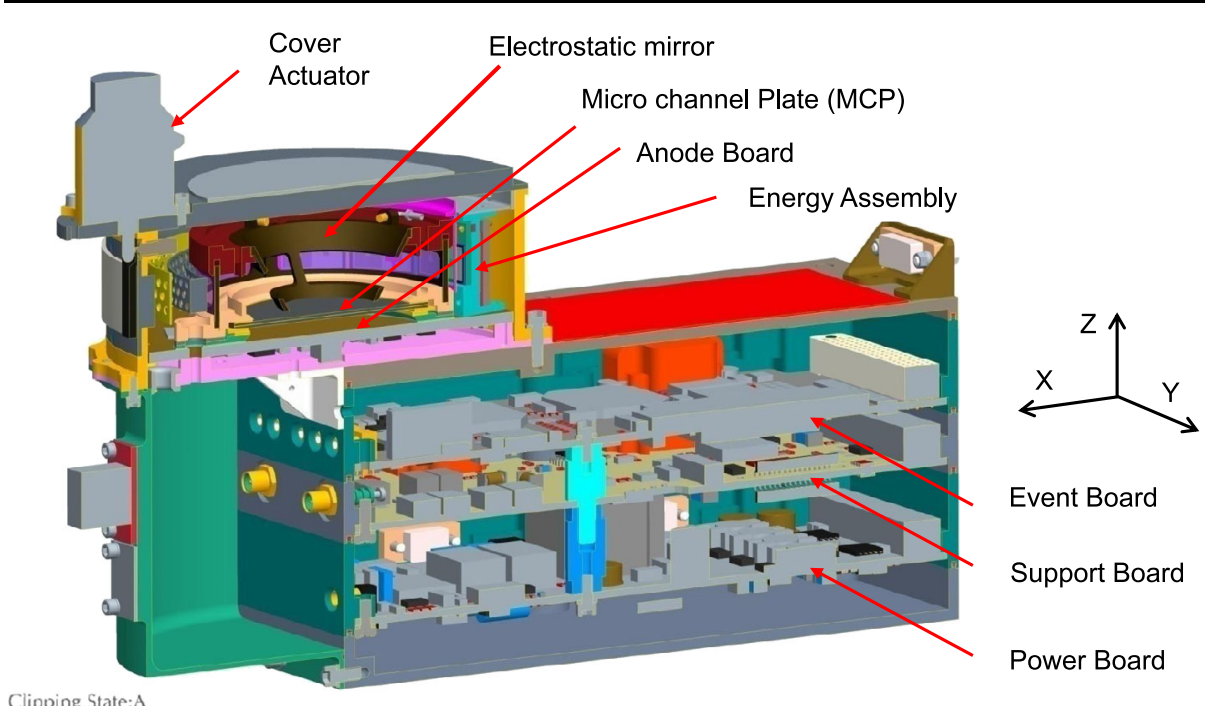

Fig. 17 The internal mechanical configuration of one of the EIS instruments
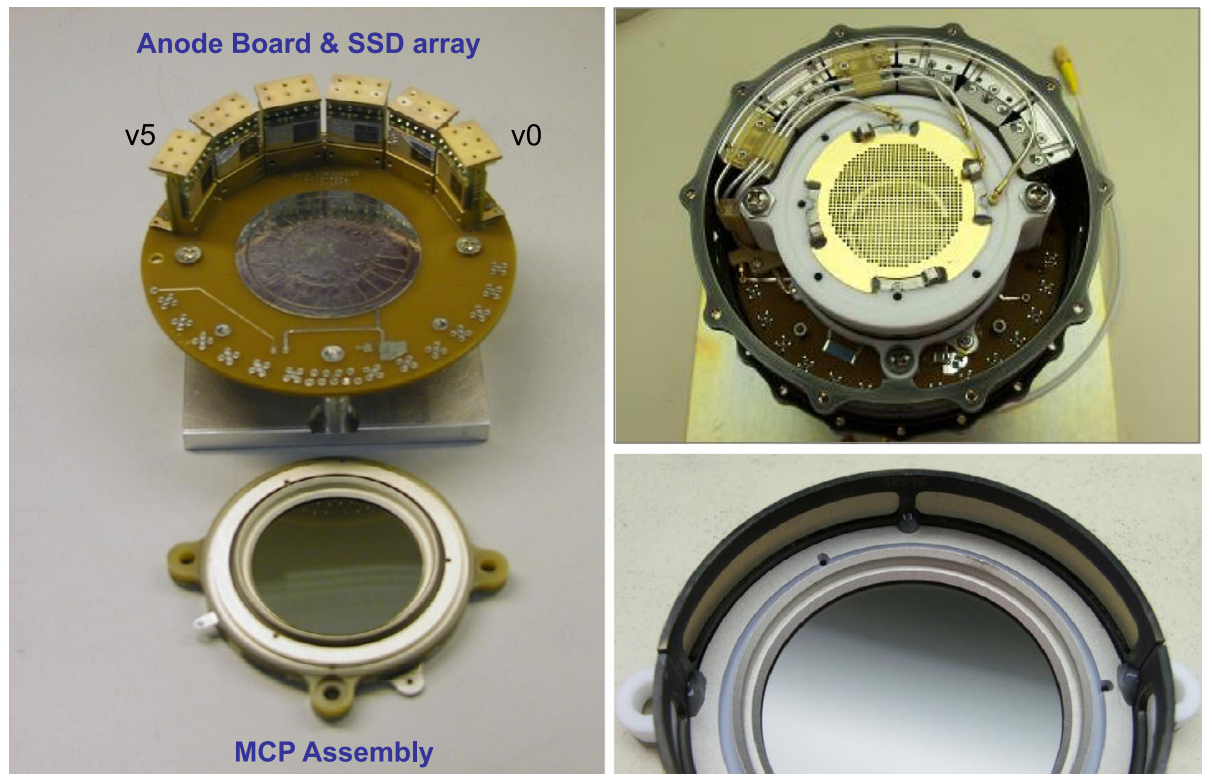

Fig. 18 Photographs of various internal components of one of the EIS sensors

on to it. The metalized anode itself in the center shows 12 anode pads in the "start" portion (bottom) and 12 anode pads in the "stop" region. The anode pads are paired to generate 6 positions in the processing of the time delay along the string of anode pads. The TOF/MCP assembly in the upper right of Fig. 18 has top and bottom Macor ceramic pieces (white in the figure) that sandwich together to hold the start and the stop frames and foils (Fig. 18 lower right). Technical specifications of the foils are given in the Appendix. 
Fig. 19 The central (3rd blade out of 5), removable blade from the EIS collimator. Mounted like a sandwich between the outer and inner shell of this removable blade is the collimator foil mounted on a stainless steel grid (visible through the holes in the outer shell)

Fig. 20 One of 6 Solid State Detector (SSD) hangers in each EIS instrument showing the mounted SSD with 4 pixels. Figure 18 shows how these hangers are mounted within the instrument
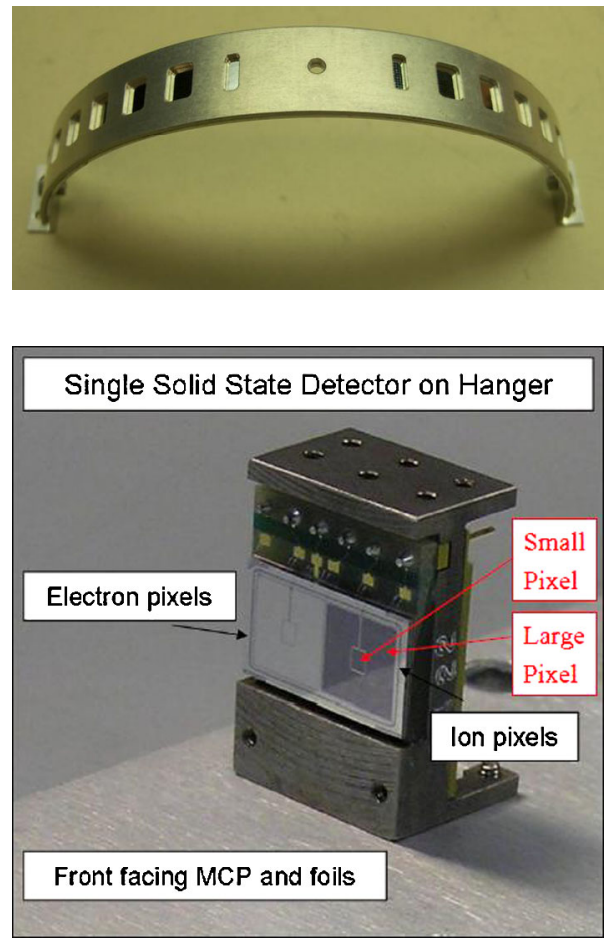

The collimator (Fig. 10, one blade of which is shown in Fig. 19) fits into the gap that is apparent in the bottom portion of the sensor assembly shown in the upper right of Fig. 18. The collimator consists of 5 blades of aluminum, each with an array of square slots (Fig. 19). The middle blade holds the collimator foil, which can be seen through the holes in Fig. 19. The sizes of the holes on each blade are graded according to distance of the blade to the center of the symmetry axis of the cylindrical sensor volume (Fig. 10). Further technical specifications are given in the Appendix.

\subsection{EIS Detectors}

Solid State Detectors (SSD's) One of 6 of the SSD holders per EIS instrument is shown in Fig. 20. The side of the holder that is shown holds a single SSD, manufactured by Canbarra, with 4 pixels, 2 electron pixels and 2 ion pixels. Each large pixel is about $0.40 \mathrm{~cm}^{2}$ and each small pixel is about $0.02 \mathrm{~cm}^{2}$, yielding sensitivity ratio of about 20 . However, once again, for the primary MMS target regions, the small pixels will likely not be needed or utilized. The electron pixels are covered with an aluminum flashing 2 microns thick. GEANT4 simulations show that, with $20 \mathrm{keV}$ discrimination on the SSD output, electrons with energy starting at about $25 \mathrm{keV}$ and above can be measured, whereas protons with energy of $250 \mathrm{keV}$ and above can to be detected. The solid state detector is 500 microns thick with a dead layer, relevant for the ion side, of about 500 Angstroms. The hanger itself is made of Aluminum and is $0.25 \mathrm{~cm}$ thick. It represents one part of the effort to shield the SSD's from most directions with $0.5 \mathrm{~cm}$ Aluminum for background control. On the back side of the hanger is a small board that contains the Energy ASICS described in Sect. 4.3. How the SSD hangers are mounted into the array that is needed for the EIS sensor is shown in Fig. 18 (upper left). 
Microchannel Plates (MCP) The single microchannel plate stack (MCP) within each EIS (Fig. 18, lower left) comprises two $5 \mathrm{~cm}$ diameter circular plates mounted, with a small gap between them, in the chevron configuration. The stack is operated with a total potential drop of between 1900 and 2400 volts, and is used with a gain of several $\times 10^{6}$. The cloud of electrons coming out of the stack is collected by a segmented anode (Fig. 18 upper left), with 12 segments in the "start" region (connected with time-delay inductors), and 12 segments in the "stop region (similarly connected). The gap between the bottom of the MCP stack and the anode is $\sim 0.25 \mathrm{~cm}$, and the potential difference that collects the electron cloud is $\sim 100$ volts.

\subsection{EIS Internal Operations, Operational Modes, and Data Products}

Although the EIS instrument can be complicated to run, for MMS it is run in a relatively simple manner, utilizing only a few basic modes of operations. When power is first applied to the EIS instrument, its processor runs software based in a fuse-link PROM resulting in the Boot Mode which establishes basic communication with the spacecraft and runs a small subset of the operating software. Other operational modes are the Cover Test Mode, the Application Mode, Cover Release Mode, HV On Mode, HV Air Safe Mode, Safe Mode, and the Science Modes. The primary EIS science modes are Calibration, Fast Survey (during which both Fast Survey and Burst data is generated), Slow Survey, and Slow Survey Electron Only.

The EIS software divides each spacecraft spin into 32 evenly spaced sectors, which corresponds roughly to 2/3 second, given the $\sim 20$ second spin period of each MMS spacecraft (note that most of the MMS instruments are "time based" whereas both EIS and FEEPS are "spin based"). As the spin rate varies, the duration of a sector varies accordingly. The CIDP sends a sun pulse to EIS every spin. Between sun pulses, the CIDP sends 5759 delta phi timing pulses. The EIS software counts delta phi pulses to space out the sectors. The spin starts, i.e. sector 0 starts, at an offset from the sun pulse. The offset, specified in delta phi units, is an up-loadable parameter. Each sector is further divided into three subsectors. The first subsector is long, 1/2 of a sector. The last two subsectors are short, 1/4 of a sector each.

The sensor hardware can be placed in a different mode during each subsector. There is a fixed dead-time, $\sim 3.8 \mathrm{~ms}$, for switching between hardware modes and the pattern of modes in each subsector is commandable (the elements are "ion-species"," ion spectra-used as a diagnostic mode", and "electron spectra"). Any subsector may collect data in any mode. Each pattern collects different data in different proportions. For example, setting subsectors 1 and 2 to ion species mode and subsectors 3 to electron spectra mode collects ion species $3 / 4$ of the time and electrons the rest of the time; ion energy mode is not collected at all.

During the Fast Survey Mode, Burst data is sampled at the full sectoring cadence of the instrument (32 sectors per spin, $\sim 2 / 3 \mathrm{sec}$ accumulation), and Survey Data is averaged to 8 samples per spin, with accumulation periods of order $2.5 \mathrm{sec}$. Slow Survey products sample in the same way as Fast Survey products, but data is accumulated only once every 10 spins. The various instrument modes, which are invoked via the stored macros, are variations on the main Application Mode; they vary only in which telemetry products are enabled and how often they are downlinked.

The EIS divides the Slow Survey period into two modes; the first, Slow Survey, is basically the same as the Fast Survey, except that we only send down science data from every tenth spin. However, when the spacecraft is inside of a specified Earth radial position (6 to 7 $\mathrm{RE})$ the instrument will start experiencing very high count rates in a region that is outside the mission's region of interest. The EIS will therefore be operated in the Slow Survey Electron 
Table 6 Onboard data products per hardware mode

\begin{tabular}{|c|c|c|}
\hline Electron energy & Ion energy & Ion species \\
\hline $\begin{array}{l}\text { Electron energy } \\
\text { spectra }\end{array}$ & Ion energy spectra & $\begin{array}{l}\text { Proton (and non-proton) } \\
\text { rates }\end{array}$ \\
\hline $\begin{array}{l}\text { Basic rates } \\
\text { (electron energy) }\end{array}$ & $\begin{array}{l}\text { Basic and diagnostic rates } \\
\text { (ion energy) }\end{array}$ & $\begin{array}{l}\text { Basic and diagnostic } \\
\text { rates (ion species) }\end{array}$ \\
\hline \multirow[t]{2}{*}{$\begin{array}{l}\text { Raw event data } \\
\text { (electron energy) }\end{array}$} & $\begin{array}{l}\text { Raw event data } \\
\text { (ion energy) }\end{array}$ & $\begin{array}{l}\text { Raw event data } \\
\text { (ion species) }\end{array}$ \\
\hline & & $\begin{array}{l}\text { Priority event data } \\
\text { (ion species) }\end{array}$ \\
\hline
\end{tabular}

Only mode. In this mode, the high voltages will be stepped down to low levels to conserve MCP lifetime and avoid the very high count rates expected in the inner belts. Only electron data products will be collected during this time.

Onboard Data Products Table 6 shows the data products that are generated for each of the 3 kinds of sub-sectored data accumulation periods (Electron Energy, Ion Energy, and Ion Species). Most of the data generated by EIS comprises a multiplicity of count accumulation channels. For the electron energy mode and the ion energy mode, the EIS software sorts the SSD energy parameter into a one-dimensional array of numbers that represent the electron or ion energy spectra, with a large number of spectral bins for "high resolution" spectra and a smaller number of spectral bins for "low resolution" spectra. For the ion species mode, one 2-dimensional TOF $\times \mathrm{E}$ array is used for events that have both a TOF and an energy to sort the events according to mass and energy (which divides the occupied regions of the TOF $\times$ Energy space, like that shown in Fig. 13 left, into a large number of discrete boxes to form the different channels; see an example in Mauk et al. 2013, Fig. 25). Another 2-dimensional array, an example of which we show explicitly here in Fig. 21, is used for events that have only TOF information (TOF and Pulse Height) to similarly sort the events according to mass and TOF (or equivalently Energy/nucleon; See Fig. 13, right).

The EIS instrument also generates what are called "Event Words" that comprise Table 4 information (or a subset of Table 4 information) about a small fraction of individual particle events that are detected by the instrument. The raw event data allows us to build (over several hours, given limitations in telemetry) displays on the ground like the two bottom panels of Fig. 13 (EIS examples are shown in Sect. 5-Figs. 23, 25, and 26). In order to diagnose all regions of the $\mathrm{TOF} \times \mathrm{E}$ and $\mathrm{TOF} \times \mathrm{PH}$ arrays, the events that are telemetered to the ground can be selected (by setting a command parameter) according to a rotating priority scheme that cycles through (with highest priority) different broad regions of $\mathrm{TOF} \times \mathrm{E}$ and $\mathrm{TOF} \times \mathrm{PH}$ arrays.

Diagnostic and Test Support EIS can be commanded into a calibration mode that injects pulses into the preamps of the TOF start, TOF stop, and SSDs to validate the fidelity and stability of the chain of circuits that process the event pulses. Both a start and a stop pulse are generated. The rate and the start-to-stop delay are set by command. The start pulse can be sent to TOF start; the stop pulse can be sent to TOF stop and the SSDs. The TOF start, TOF stop, and SSD pulses can be enabled or disabled individually by command. The EIS hardware can be commanded to measure SSD energy channel or MCP pulse height baseline values instead of doing its normal event processing. The results appear in the event FIFO with the relevant information shown in Table 4. 
Fig. 21 The EIS

Time-of-Flight $\times$ Pulse-Height $(\mathrm{TOF} \times \mathrm{PH})$ channels as they are configured in the EIS-internal $\mathrm{TOF} \times \mathrm{PH}$ data number matrix. The gap between the protons and the heavy ions is intended to minimize the mixing of species. This matrix can be adjusted by uploading a single parameter that rescales the horizontal axis, or by uploading new tables altogether

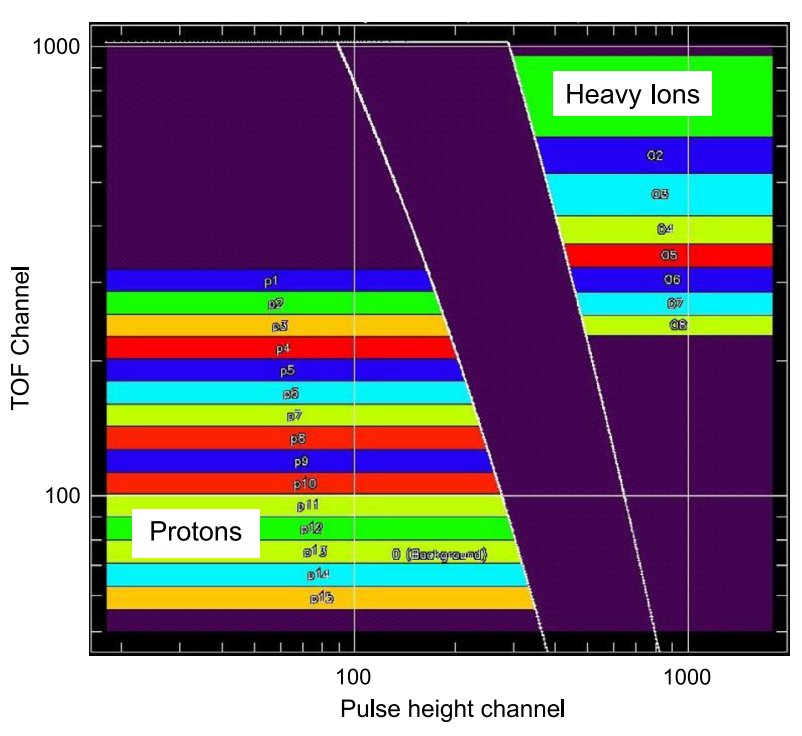

Fig. 22 The relative measurement efficiency of EIS detection of various particle species as a function of incoming total energy

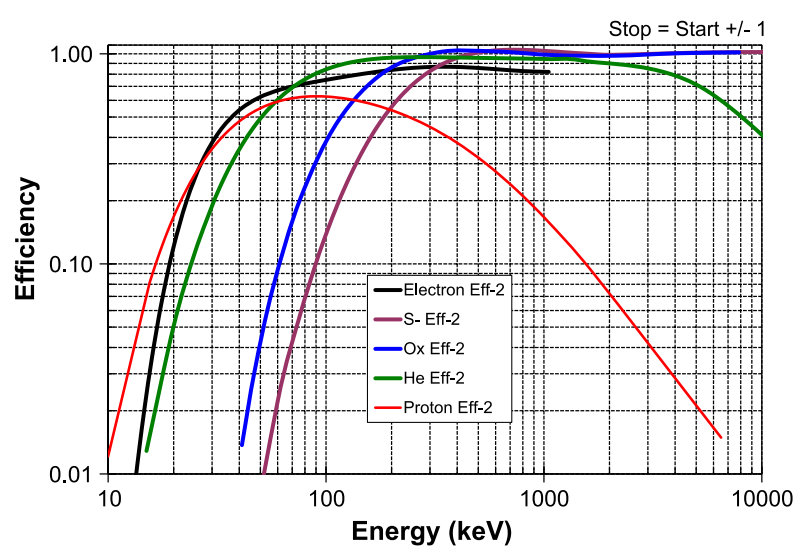

\section{EIS Calibration and Performance}

\subsection{Efficiencies, Channel Characteristics and the Factors that Affect Them}

The efficiency by which the typical EIS instruments measure electrons, protons, helium ions, and oxygen ions as a function of incoming energy (keV) is shown in Fig. 22. (Sulfur is also shown, revealing the JEDI heritage for this plot.) The roll-off in ion efficiency as one goes from intermediate to lower energies occurs primarily as a result of scattering; particles come into the collimator on valid trajectories but scatter in the collimator foil or the active start foil, change their directions of flight, and subsequently strike a non-sensing part of internal sensor volume. Note that the determination of the entrance direction is done at the start anode for ions, and so the subsequent scattering does not affect the determination of the angle of arrival. The roll-off in electron efficiency as one goes from intermediate to lower energies also has a scattering component to it, but is dominated by the electron interactions with the $2 \mathrm{~mm}$ aluminum flashing on the electron sensors. The ion roll-off in efficiency as 
one goes from intermediate energies to very high energies for protons and helium ions occurs as a result of the reduction in the efficiency for the generation of secondary electrons within the start foil and the stop foil. The efficiency of secondary electron generation for ions (the foils are not used to detect the electrons) scales roughly as the stopping power ( $\mathrm{dE} / \mathrm{dx}$; often characterized with the units $\mathrm{keV} /$ micron), and the stopping power of protons and helium ions fall substantially for higher energies. The total sensitivity for measuring a specific species at a specific energy is determined by the efficiency $(\epsilon)$ times the geometric factor $\left(\epsilon \cdot \mathrm{G}\right.$; where $\mathrm{G}$ has the units $\mathrm{cm}^{-2} \mathrm{sr}^{-1}$; see Appendix for values). Specifically, Intensity (I: particles $\left.\mathrm{cm}^{-2} \mathrm{~s}^{-1} \mathrm{sr}^{-1} \mathrm{keV}^{-1}\right)=\mathrm{R} /\left[\epsilon \cdot \mathrm{G} \cdot\left(\mathrm{E}_{2}-\mathrm{E}_{1}\right)\right]$, where $\mathrm{R}$ is rate (counts/sec) for a specific channel and $E_{2}$ and $E_{1}$ are the energy boundaries of specific energy channel. One of the trade-offs that may be made with the setting of an on board parameters is the degree to which the start sector matches the stop sector (the start and stop sectors may not match due to scattering). One may require that "stop $=$ start $\pm n$ ", where " $n$ " may be 0,1 or 2 . For $\mathrm{n}=0$ the measurements are the cleanest and with the lowest time dispersion. For high values of $n$ the efficiency is greater, but the sensitivity to accidentals (Sect. 3.3) is also greater. Figure 22 was made under the assumption that $n=1$.

The efficiency curves shown in Fig. 22 are primarily based on simulations grounded with empirical information. These curves are being and will be verified and corrected, using two approaches: (1) Ground cross-calibration procedures described at the end of Sect. 5.2, and (2) Flight cross calibration activities described in Sect. 5.5.

\subsection{Calibration Procedures and Facilities}

The response of the EIS instruments is complicated, and our understanding is based on a coordinated array of approaches, specifically: (i) bench testing of channel gains and other characteristics based on calibrated pulse inputs, (ii) calibrations using particle accelerator beams, (iii) calibrations using radiation sources, (iv) simulations of particle interactions with matter using such tools as GEANT4, and (v) geometric calculations. Two particle accelerators were used for calibrating EIS: (1) the JHU/APL ion particle accelerator that generates narrow ion beams of $\mathrm{H}, \mathrm{He}, \mathrm{O}$ ( $\mathrm{N}$ often used as proxy), Ar, and other ions species from energies as low as about $12 \mathrm{keV}$ up to $170 \mathrm{keV}$; and (2) the GSFC Van de Graff, accelerator that generates electron and ion species beams from $\sim 100 \mathrm{keV}$ to $>1 \mathrm{MeV}$. Two different radiation sources were used. These sources are a Barium Ba133 source and a degraded Americium Am241 radiation source (the source is degraded by placing a thin mylar foil between the source and the sensor, which yields a very broad spectrum of alpha particle energies). To perform the calibrations we have procured sources that are configured so as to completely fill the fieldsof-view of the EIS sensor (we call these sources the "Geordi" sources given that they look much like the artificial eyes worn by Geordi La Forge in the television show: Star Trek, The Next Generation). Because the sources fill the EIS field of view, all 6 look directions are calibrated simultaneously.

The Ba133 source provides the information needed to convert internal SSD energy data numbers (dn-E) into energy ( $\mathrm{keV}$ ) for all 24 SSD pixels (6 large ion pixels, 6 small ion pixels, 6 large electron pixels, and 6 small electron pixels). A typical Ba133 spectrum from the EIS sister instrument is shown in Mauk et al. (2013; Fig. 29 of that paper).

The degraded AM241 provides a broad energy-distribution alpha source that tests the Time-of-Flight system and energy system simultaneously for all $6 \mathrm{TOF} \times \mathrm{PH}$ look directions, all 6 of the TOF $\times$ E large-pixel look directions, and all 6 of the TOF $\times$ E small pixel look directions. Typical degraded Am241 TOF $\times$ E spectra are shown in Fig. 23, with a large pixel to the left and a small pixel to the right. Here we see that essentially the entire 


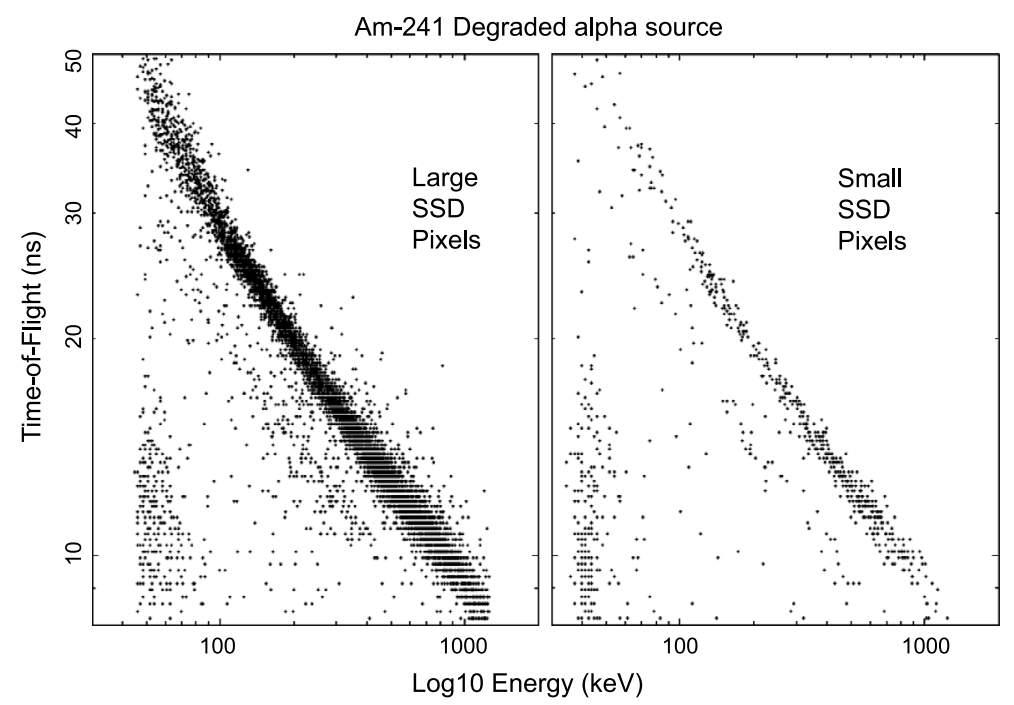

Fig. 23 The response of the EIS TOF $\times$ E system to a degraded AM241 alpha-particle radiation source. The $\sim 5 \mathrm{MeV}$ emitted alpha particles are degraded (and spread over a broad range of energies) with a mylar foil. This plot tests the response of both the energy and the TOF system and, by bootstrapping the Ba133 calibrations, allows one to determine the conversion from TOF data number to TOF in nsec for the faster times of flight. The left panel is for a large SSD pixel, and the right panel is for a small SSD pixel, showing that the small pixels provide equivalent information at reduced sensitivity

energy range is tested and the shortest, most challenging portion ( $\sim 5$ to $\sim 50 \mathrm{nsec})$ of the full EIS TOF range ( $\sim 5$ to $\sim 160 \mathrm{nsec})$ is also tested. These spectra test uniformity across all look directions and all units, and provide a backup method (alternative to accelerator beams) of determining how to convert internal TOF data numbers (dn-TOF) into true TOF (nsec) by bootstrapping off of the Ba133 determination of the true energies. The ghost signatures in the TOF $\times \mathrm{E}$ displays are due to a combination of scattering of ions within the sensor (particularly off of the cone-like electrostatic mirror structure), SSD edge effects, and high energy penetrations of grids.

One of the calibration activities that is still ongoing is to use the degraded AM241 Geordi source as a "standard candle" for cross-calibrating EIS and FEEPS. The Geordi source completely fills the fields of views of both EIS and FEEPS with a relatively flat (in energy) alpha spectrum. These runs determine the relative energy-dependent efficiency of EIS and FEEPS (and we are also looking to use the same standard candle to cross calibrate the energy overlap regions with the plasma sensors.)

\subsection{EIS Performance Verification}

In this section we show or cite selected exhibits that we used to verify the performance of the EIS instrument design. Many of these exhibits were taken from the calibration of the sister JEDI instruments, with the AM241 calibrations like those shown in Fig. 23 use to verify that the performance of each EIS unit is the same.

Figure 24 shows $\mathrm{TOF} \times \mathrm{PH}$ results for $\mathrm{N}+$ (left; proxy for $\mathrm{O}+$ ) and $\mathrm{H}+$ (right). These and similar displays demonstrate that EIS satisfies the requirement of measuring heavy ion energies as low as $45 \mathrm{keV}$ and protons for energies as low as $20 \mathrm{keV}$. Note that the splitting of 

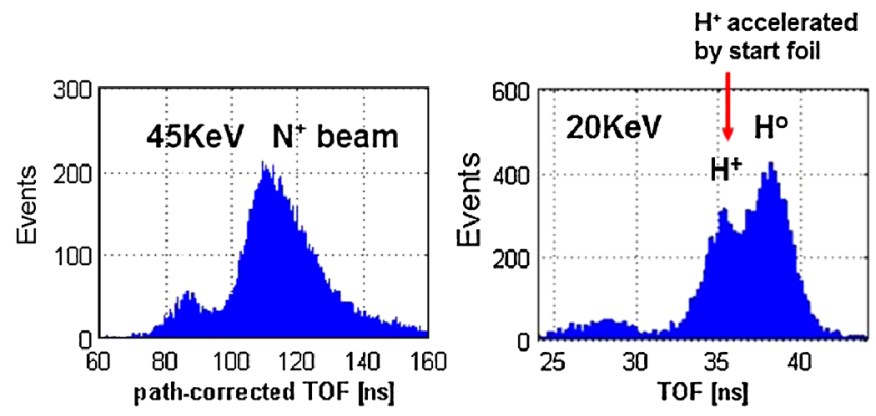

Fig. 24 TOF $\times$ Pulse-Height event distributions for an incoming $45 \mathrm{keV} \mathrm{N}+$ beam (left) and an incoming $20 \mathrm{keV}$ proton beam on the right. The $\mathrm{H}+$ and $\mathrm{H} 0$ peaks on the proton display result from charge redistribution in the collimator foil and the subsequent acceleration of the $\mathrm{H}+$ (not the $\mathrm{H} 0$ ) by the $\sim 2.5 \mathrm{kV}$ that participates with the secondary electron optics. The lowest energy peaks (centered on $85 \mathrm{~ns}$ on the left and 28 ns on the right) are contaminations resulting from the primary particles striking an edge of the electrostatic deflection mirror (Fig. 12) with the subsequent secondary electrons finding their ways to the MCP. The exact shape of that peak during calibrations depends on the details of the accelerator beam alignment
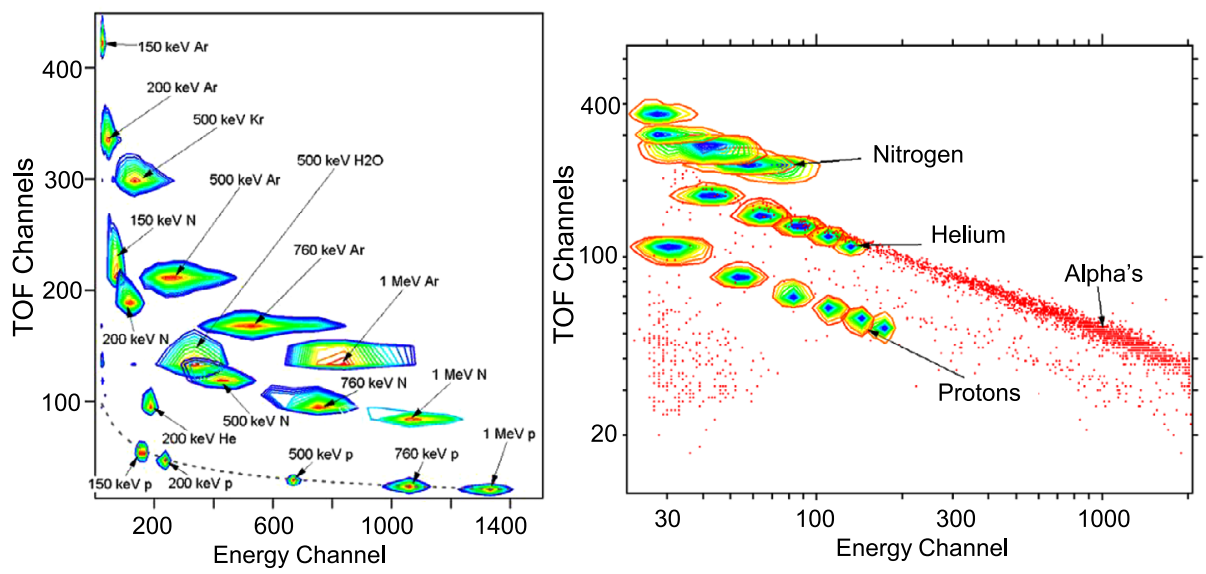

Fig. 25 TOF $\times$ E calibration runs obtained by the high energy ion accelerator at GSFC $(l e f t)$ and the lower energy JHU/APL ion accelerator (right). This figure shows a portion of the broad range of energies detected by EIS and that for TOF $\times \mathrm{E}$ the elemental species are well separated. On the right panel, the slight shift between the accelerator measurement of helium and the degraded Am241 source measurement of alpha particles is a result of slight differences in the data-number (DN) to energy conversion for different SSD chains. These differences in DN to energy conversion are accounted for in our calibration matrices

the proton response into $\mathrm{H}+$ and $\mathrm{H} 0$ is due to charge redistribution as the protons penetrate the collimator foil.

Figure 25 shows 2 different Time-of-Flight $\times$ Energy $(\mathrm{TOF} \times \mathrm{E})$ displays, one from the GSFC ion accelerator facility (left) and one from the JHU/APL ion accelerator facility, that demonstrate (together with Fig. 24) that the required ion energy ranges are achieved and that the $\mathrm{TOF} \times \mathrm{E}$ function achieves the mass discrimination capabilities that are required. For the display on the right, a degraded Am241 spectrum (alpha particles) is shown in addition to the accelerator beam results. The slight shift between the Am241 and beam responses is 

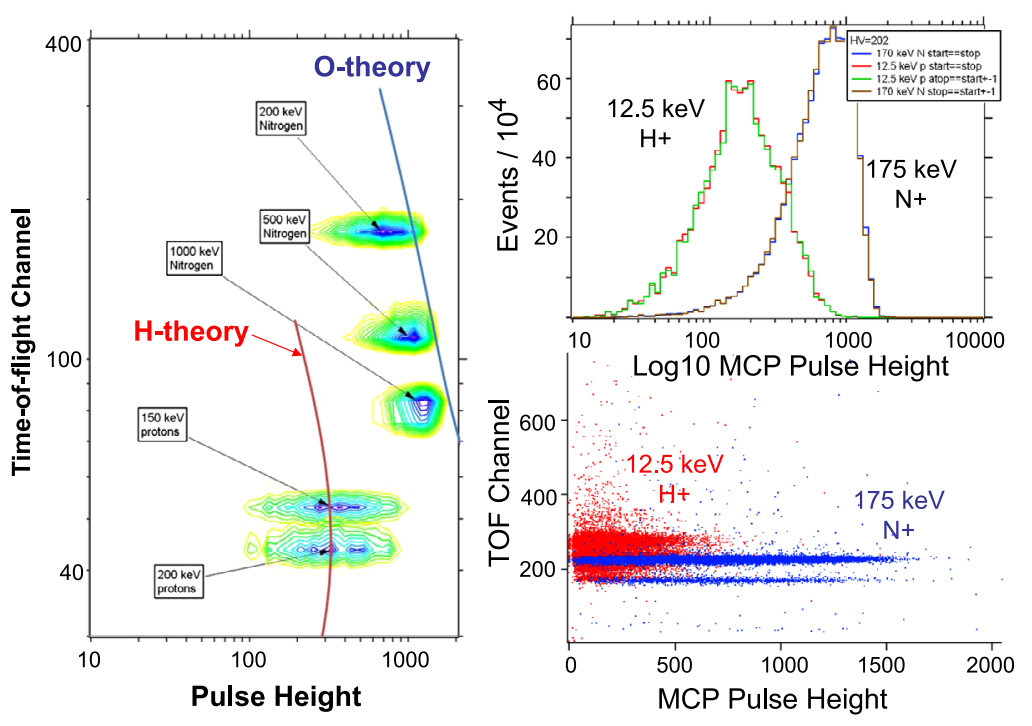

Fig. 26 TOF $\times$ PH calibration runs obtained by the high energy ion accelerator at GSFC (left) and the lower energy JHU/APL ion accelerator (right). TOF $\times \mathrm{PH}$ measurements do not separate mass species to the degree that TOF $\times \mathrm{E}$ measurements do, but these measurements show that EIS meets requirements. Clean measurements are obtained by eliminating the measurements in the regions of overlap (e.g. see Fig. 21), at the price of reduced sensitivity

due to variations in the data-number-to-energy conversions for different solid state detector chains.

Figure 26 shows that the Time-of-Flight $\times$ Pulse Height $(\mathrm{TOF} \times \mathrm{PH})$ function separates light ions (protons) from heavy ions (nitrogen is used as a proxy). This function is used for the lowest energy ion measurements. The separation is not (and is not expected to be) nearly as clean as the separation that is achieved with the TOF $\times \mathrm{E}$ function. The approach that is taken for in-flight measurements is to sample the heavy ions only at the highest pulse height region of the distribution in order to get a good sampling of the heavy ions uncontaminated with protons. Figure 21 shows how that sampling is achieved with the onboard channel tables (the regions just between the proton and heavy ion channels are not sampled), and the gap size can be modified (with new tables) as we gain experience with each unit.

Figure 27 shows that EIS achieves its angle resolution requirements (the capabilities shown combined with the geometric calculations for the collimator prove that EIS achieves the $30^{\circ}$ resolution goal). The top panel shows the $6 \mathrm{TOF} \times \mathrm{PH}$ view directions as they are rotated across an accelerator beam. The bottom panel shows the $6 \mathrm{TOF} \times \mathrm{E}$ view directions as they are similarly rotated across an accelerator beam. The TOF $\times$ E measurements show a narrower response because the ion portion of the SSD's that participate with the TOF $\times \mathrm{E}$ measurements cover only half the backplane (Fig. 20). Note that the determination of the arrival direction of the ions is performed at the start position, eliminating the subsequent scattering as a cause for smearing out of the angular response.

The performance verification of other aspects of EIS can be found by examining exhibits generated for the EIS sister instrument JEDI (JEDI: Mauk et al. 2013). Examples included energy resolution (JEDI Fig. 34; JEDI Fig. 36), the fact that efficiency scales with the particle stopping power (dE/dX; JEDI Fig. 35), and rate saturation effects (JEDI Figs. 38 and 39) 
Fig. 27 JHU/APL ion

accelerator calibrations showing that the EIS TOF $\times \mathrm{PH}$ (top) and the TOF $\times \mathrm{E}($ bottom) achieve the required angular resolutions. The six peaks are from the 6 EIS look directions as the EIS instrument is rotated across the beam. The test beam here comprised $\sim 100 \mathrm{keV}$ protons. Because the arrival direction of the ions is determined exclusively using the start signal for ions, subsequent scattering does not affect the angular response (only the efficiency of detection), and so this angular response for ions is very insensitive to ion energy or species

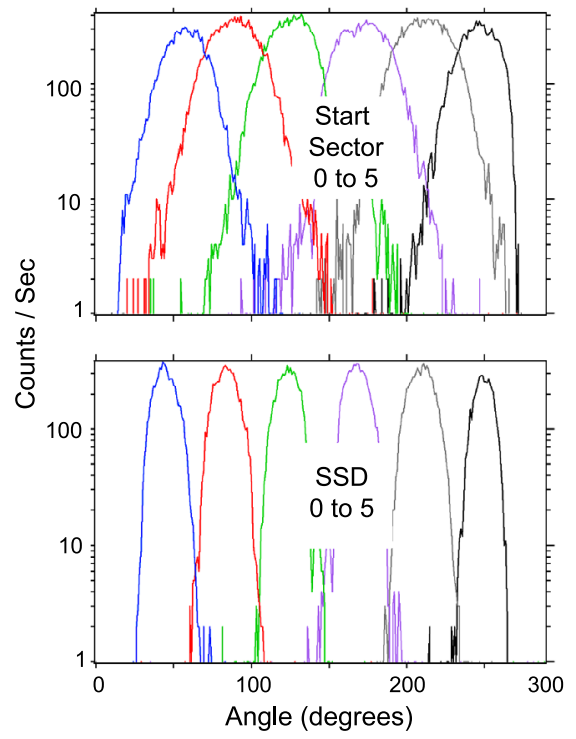

\subsection{EIS Data Features}

There are "ghost" features within the JEDI data. Figure 24 (left) shows one such feature. The small bump that resides just below the main peak for this TOF $\times \mathrm{PH}$ measurement is the result of the primary particle striking an edge of the electrostatic mirror (Figs. 12 and 17), with the generation of secondary electrons that find their way to the MCP (the right-hand panel for $20 \mathrm{keV} \mathrm{H}+$ shows a corresponding featured centered on about 27-28 ns). A similar looking feature is apparent in the TOF $\times \mathrm{E}$ spectrum shown in Fig. 23 for both large and small pixels (the ghost track), resulting from SSD edge effects. There are a scattering of other events well below the main track that result from penetrations of or scattering off of stainless steel grids that hold the various foils within the sensor.

\subsection{In Flight Calibration Processes}

The EIS instruments have an inflight calibration mode described in Sect. 4.6. Our plan is to invoke this mode periodically (once per quarter).

For each of the different species products (these include including TOF $\times \mathrm{E}$ and TOF $\times$ PH, electron spectra, and ion spectra) each EIS sensor comprises 6 different views. We determine the relative precision between these multiple views by analyzing EIS data within regions where the particle distributions are roughly time-stationary, homogeneous, and isotropic (nominally closer to Earth than the science target regions; Sect. 6). Differences in the responses of the different EIS views can result from non-uniform gains on the MCP, slightly different channel definitions because of variations between the same components on different instruments or view directions, differences in discrimination levels due to differences in detector noise characteristics, etc. Analysis of the differences in view direction responses can be mitigated by adjustments on the ground to calibration matrices (geometric factors, gain factors, etc.) and adjustments onboard (discrimination levels, MCP bias voltages, new table uploads, etc.). During orbital operations such inter-comparisons can be performed every orbit using nominal Slow Survey science data (depending on geomagnetic 
conditions), but special periods of FAST SURVEY with BURST data cross calibrations will be performed occasionally (Sect. 6).

These special calibration periods will also be used to cross-calibrate EIS and FEEPS (and also the energy overlap regions of the plasma sensors). We are proposing to do this by running the sensors for a few minutes every couple of weeks at radial distances of 7$8 \mathrm{RE}$ in their Fast Survey and Burst modes. In these regions the particle distributions are more likely to be isotropic, homogeneous, and time stationary in the spacecraft frame; thus more suitable for comparing relative responses. It is understood that nature will not always cooperate, but over time a good cross comparison should be achieved.

A principle concern is determining and setting the efficiency of detection of secondary electrons coming from the start and stop foils, given the changing gain states of the MCP's over time. There are two features of EIS that make this process much easier than it has been on heritage instrument (prior to JEDI). The first feature is that complete detailed pulse height distributions (2048 channels) can be obtained in flight for the start region of the MCP, allowing the detailed response of the start system (start foil, MCP, anodes) to incoming particles (e.g. Fig. 26). To take advantage of this capability, the so-called event data must be telemetered to ground. The complete diagnostic event data (Table 4) can be sent, but generally to preserve telemetry volume a subset of that information is sent. After several hours of sampling, the individual event data may be sorted according to energy, TOF, PulseHeight (PH), and look direction, and so the PH distribution for a "standard candle" energy (e.g. $100 \mathrm{keV}$ protons) for each look direction can be generated and compared with ground distributions and with other distributions in space. Because the pulse height distribution is obtained only for the start pulses, this procedure only diagnoses the evolution of secondary electron detection of the start region, not the stop region. However, the start region is where we expect the more rapid changes in efficiency due to the great flux of particles and UV light onto the start foil than is expected on the stop foil (the geometric factor of just the start foil is a factor of 3-4 greater than the geometric factor of just the stop foil).

The second feature that EIS contains to determine the efficiencies of secondary electron detection, for both the start and the stop regions, is the ability to count various kinds of coincident events. Specifically, there are counters that report coincident SSD-Start counts and coincident SSD-Stop counts. These counters can be combined to obtain the efficiencies of secondary electron generation for both the start regions and the stop regions (algorithms for this process are shown in Fig. 42 in Mauk et al. 2013).

The principal responses to changes in the efficiency of secondary electron generation are: (a) to increase the gain of the MCP by increasing its bias voltage, and/or (b) to modify the $\mathrm{TOF} \times \mathrm{PH}$ look-up tables by adjusting a multiplicative parameter.

\subsection{Flight Performance of Sister Instruments}

EIS has two sister investigations that are very close in design to that of EIS (Sect. 4.2). Both of these instruments are now in flight and taking data: Juno JEDI (Mauk et al. 2013) and Van Allen Probes RBSPICE (Mitchell et al. 2013). JEDI has taken data within the interplanetary environment showing that the instruments are performing as expected and as observed on the ground before flight (Fig. 28). Figure 29 shows JEDI data taken during the Juno Earth flyby that took place on 9 October 2013. The top panel shows electrons and protons $(\mathrm{TOF} \times \mathrm{E})$ spectrograms for positions upstream of Earth's shock and magnetopause (where escaping upstream ions were observed), through the sharp magnetopause transition, and deep into the (first) outer electron and (then) inner proton radiation belts. The bottom panel shows details very relevant to what EIS will be observing during orbit phase 1 near Earth's sunside magnetopause. Much angular structure is evident, revealing EIS's capability to sound 


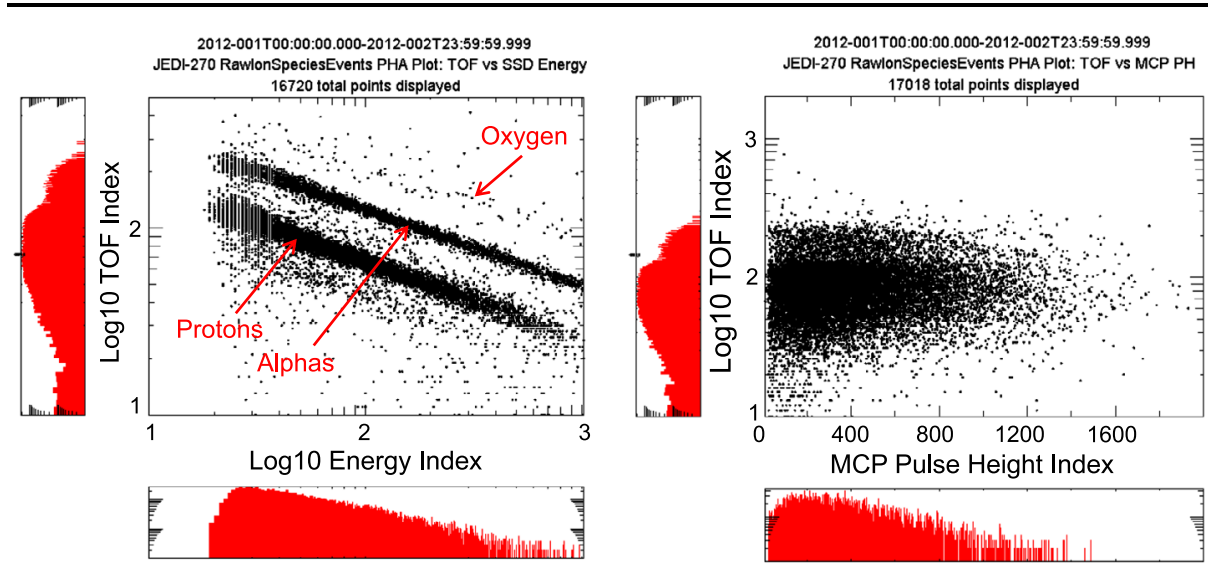

Fig. 28 TOF $\times \mathrm{E}($ left $)$ and TOF $\times$ PH spectra taken in flight by the EIS sister instrument JEDI-270 during an enhancement in the interplanetary ion intensities
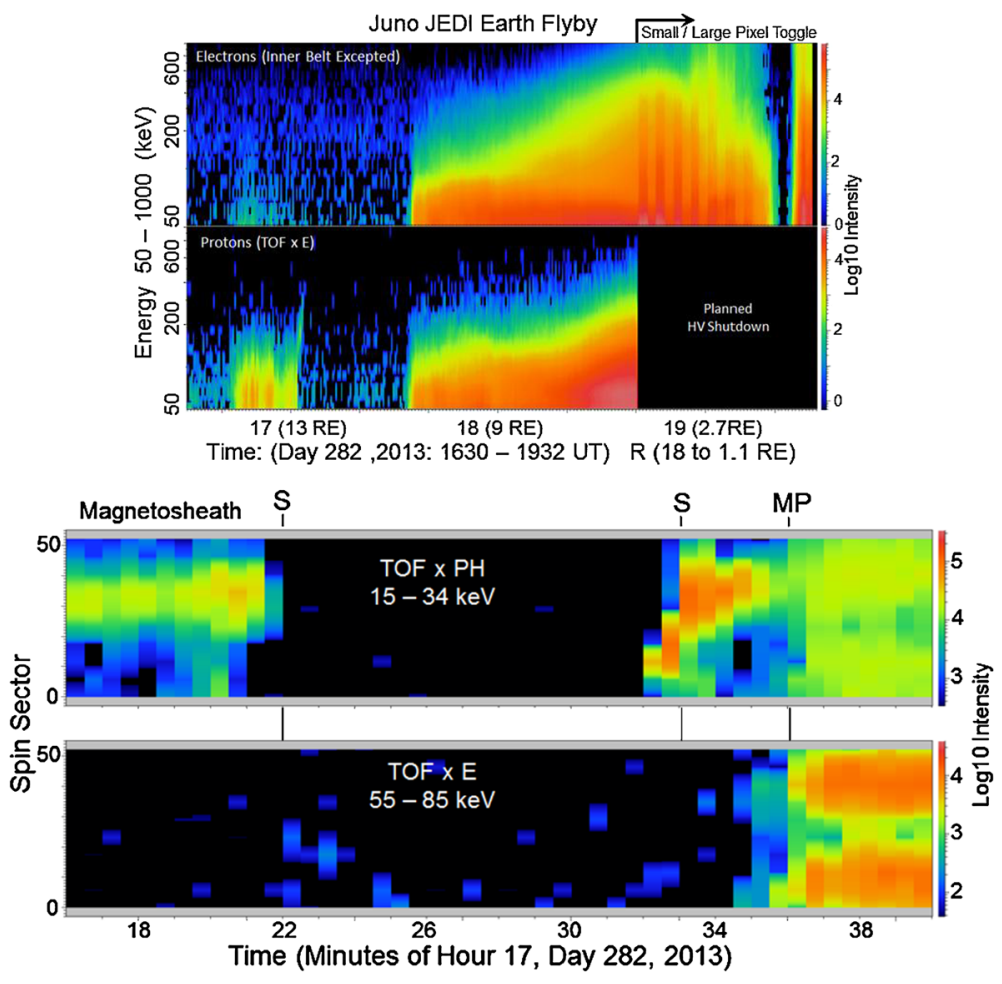

Fig. 29 Measurement made by the EIS sister instrument JEDI during the Juno encounter with Earth's magnetosphere, validating the ability of EIS to make quality measurements within one of the key target regions of MMS, the sun-side magnetopause. The identification of the magnetopause boundary (M) and bow shock crossings (S) were performed by Connerney et al. (2013) with Juno magnetometer data 


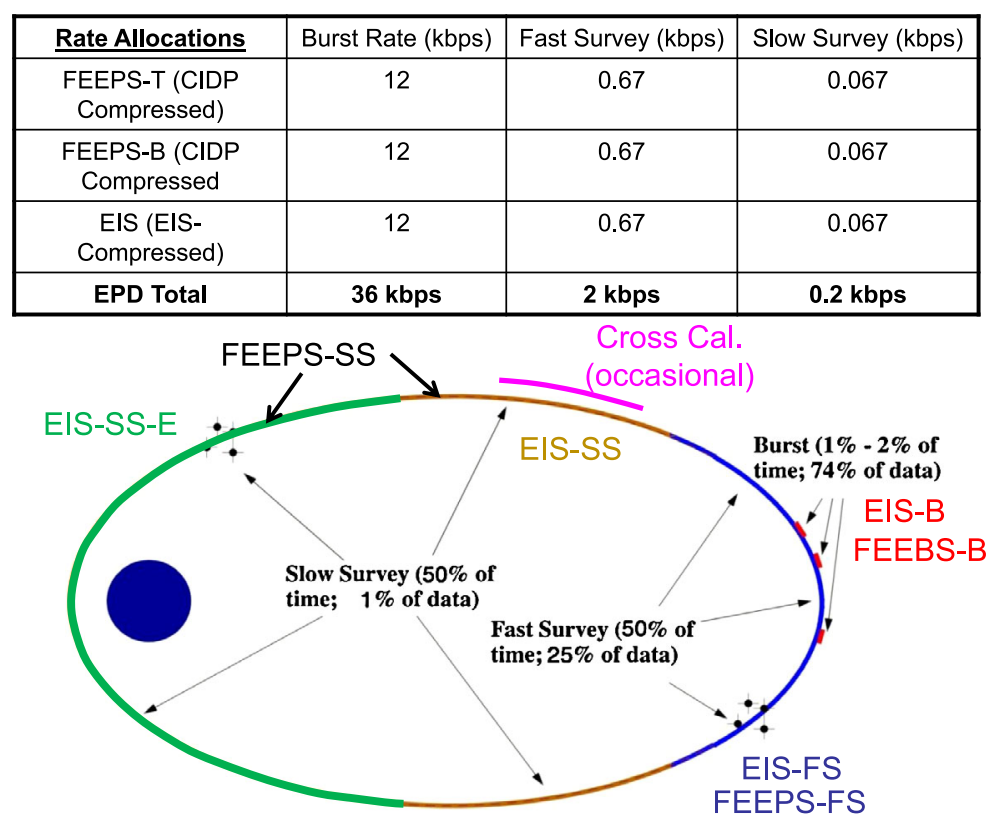

Fig. 30 (bottom) A typical MMS orbit showing the EPD modes of operation during such orbits (see the main text for a discussion of this figure). The table shows EPD data rate allocations

distant structures with anisotropy analysis. Recall also that EIS is a factor of 3 more sensitive than is JEDI.

Both JEDI and RBSPICE have suffered some hiccups with their High Voltage operations. The JEDI hiccups are associated with higher than expected UV light entering the collimator, and operating the HV when the spacecraft spin-sun angle is not within the science operations orientation. By taking more care when the HV is operated and uploading software that provides flexibility in the response to UV-induced micro-discharges within the MCP, the JEDI $\mathrm{HV}$ issues have been resolved (Mauk et al. 2013). The RBSPICE HV hiccups are apparently associated with internal charging that can occur when the instrument encounters the highly dense plasmas of the plasmasphere, with densities rising to the several 1000's. While the RBSPICE instruments are meeting all science objectives, the HV issues have caused operational complexities. Possible problems are mitigated on EIS by never operating its high voltages inside of 6 to $7 \mathrm{RE}$ (7 RE during the early phases), just as was done for the Juno Earth encounter (Fig. 29, top), where no HV problems were encountered. The densities to be operated within for EIS are much more like the densities that JEDI has and will encounter.

\section{EPD Operations}

Figure 30 shows the nominal EPD operations activities during a typical scientific orbit of the MMS spacecraft, and the data allocations for those different periods. There are nominally two modes of operation for all instruments: FAST SURVEY for when the spacecraft are in the primary science target regions (Earth's Magnetopause on the dayside and Earth's deep (> 15 RE) magnetotail on the night side), and SLOW SURVEY for providing context during other parts of the orbit. During the FAST SURVEY periods, EIS simultaneously 
generates FAST SURVEY data (EIS-FS) and BURST data (EIS-B). For EIS, the SLOW SURVEY period comprises two different modes of operation, High Voltage (HV) on (EISSS; the nominal mode), and HV off (EIS-SS-E; generating electron data products only). For FEEPS, the onboard Central Instrument Data Processor (CIDP) simultaneously generates FAST SURVEY data (FEEPS-FS) and BURST data (FEEPS-B) from the raw FEEPS data (Blake et al. this issue). During the FAST SURVEY periods for all instruments, the Burst Selection Process is located on the ground at the MMS SOC (Baker et al. this issue), which decides which brief periods during the FAST SURVEY portion of the orbit are the most interesting from the perspective of reconnection science, and decides which small fraction of the BURST data is to be telemetered down to the ground.

As indicated in Fig. 30, occasionally the EPD team will ask for a period of high rate data within the nominal SLOW SURVEY period in order to perform cross calibrations within regions of space that are nominally homogeneous and isotropic during geomagnetically inactive periods.

One kind of data product that we have not yet mentioned is the so-called Burst Quality Flags (BQF), generated on 10 second intervals for most instruments and on $1 / 2$ spin cadences for EIS and FEEPS. Table 7 shows the BQFs that are generated by EIS and for FEEPS by the CIDP. Weighted sums or averages of the BQF's from all of the instruments are used for ground-automated decisions to be made about what BURST data is to be sent down during the FAST SURVEY periods. Because reconnection simulations do not predict consensus energetic particle signatures, the EIS and FEEPS BQF's are not initially used for making BURST telemetry decisions. Only after experience is gained with these parameters, and the extent to which they characterize and summarize the detailed science data, might the EIS and FEEPS BQF's be used to make decisions about BURST telemetry.

\section{EPD Data Processing, Flow, and Archiving}

The Data Processing, Flow, and Archiving plan assumes a very close coordination between the Instrument Operations Centers and the central Science Operations Center (SOC) located at the University of Colorado LASP. Note that there are two entities at LASP that are of interest here: EPD LASP (a part of the EPD team) and SOC LASP (a mission level entity). Figure 31 shows the plan for processing the EPD (FEEPS and EIS data). Algorithms for generating Level $1 \mathrm{a}$ and Level $1 \mathrm{~b}$ data are generated by the FEEPS EPD process at LASP for FEEPS data, and at APL for EIS, and transferred to the SOC process at LASP for execution. FEEPS Level 2 data is generated by the FEEPS EPD process at LASP, but the procedures for doing so are certified by Aerospace and the University of New Hampshire. The EIS Level 2 data is generated by APL. The FEEPS and Level 2 products are transmitted to SOC process at LASP. Figure 31 shows some additional details of the processing, for example specifying the need for the availability of magnetic field data and ephemeris data at various stages of the production.

We document here only those data products that will be made easily available to the broad scientific community; Level-2 data and Level-3 (Preliminary).

EPD Level 2 Data These are observatory level data products (for each spacecraft there would be one set from FEEPS (the two instruments combined) that incorporates some EIS data, and one set from EIS). The channel contents are "vectors", specifically: \{geometricmean-energy, counts-per-accumulation, counts-per-second, and intensity \}, so that information about the cleanliness of and errors within the channels is not lost. For each record 
Table 7 EPD Burst Quality Flags (BQF's)

\begin{tabular}{|c|c|c|c|c|c|c|}
\hline Number & Quantity & Source & Spectral range & Amplitude range & Algorithm & Physical signature \\
\hline 1 & $\begin{array}{l}\text { Electron } \\
\text { intensity }\end{array}$ & $\begin{array}{l}\text { FEEPS } \\
\text { electrons }\end{array}$ & $\begin{array}{l}25-500 \mathrm{keV} \\
\text { electrons }\end{array}$ & $\begin{array}{l}\text { Indexed to } \\
0-5 \text { (TBR) }\end{array}$ & $\begin{array}{l}\text { Hemisphere average } \\
\text { on energy-integrated } \\
\text { electrons every } 1 / 2 \\
\text { SC spin }\end{array}$ & $\begin{array}{l}\text { Local intensity } \\
\text { enhancements } \\
\text { indicate local } \\
\text { acceleration. } \\
\text { Inconsistency } \\
\text { between SC indicate } \\
\text { tight local } \\
\text { environment spatial } \\
\text { structuring }\end{array}$ \\
\hline 2 & $\begin{array}{l}\text { Electron } \\
\text { variability }\end{array}$ & $\begin{array}{l}\text { FEEPS } \\
\text { electrons }\end{array}$ & $\begin{array}{l}25-500 \mathrm{keV} \\
\text { electrons }\end{array}$ & $\begin{array}{l}\text { Indexed to } \\
0-5 \text { (TBR) }\end{array}$ & $\begin{array}{l}\text { Deviation of the } \\
\text { mean of Item } \# 1 \text { of } \\
\sim 1.5 \text { sec over } 1 / 2 \mathrm{SC} \\
\text { spin }\end{array}$ & $\begin{array}{l}\text { Fast variability } \\
\text { indicates tight } \\
\text { magnetic geometries, } \\
\text { yielding fast intensity } \\
\text { changes possibly } \\
\text { associated with } \\
\text { reconnection }\end{array}$ \\
\hline 3 & $\begin{array}{l}\text { Electron } \\
\text { anisotropy }\end{array}$ & $\begin{array}{l}\text { FEEPS } \\
\text { electrons }\end{array}$ & $\begin{array}{l}25-500 \mathrm{keV} \\
\text { electrons }\end{array}$ & $\begin{array}{l}\text { Indexed to } \\
0-5 \text { (TBR) }\end{array}$ & $\begin{array}{l}\text { Ratio of selectred } \\
\text { look directions for } \\
\text { common energy } \\
\text { ranges. Maximum } \\
\text { values over } 1 / 2 \mathrm{SC} \\
\text { spin }\end{array}$ & $\begin{array}{l}\text { Electron beaming. } \\
\text { Remote sensing of } \\
\text { acceleration }\end{array}$ \\
\hline 4 & $\begin{array}{l}\text { Ion } \\
\text { intensity }\end{array}$ & EIS ions & $\begin{array}{l}25-500 \mathrm{keV} \\
\text { protons }\end{array}$ & $\begin{array}{l}\text { Indexed to } \\
0-5 \text { (TBR) }\end{array}$ & $\begin{array}{l}\text { Hemisphere average } \\
\text { on energy-integrated } \\
\text { protons every } 1 / 2 \mathrm{SC} \\
\text { spin }\end{array}$ & $\begin{array}{l}\text { Locality of intensity } \\
\text { enhancements. } \\
\text { Acceleration. } \\
\text { Inconsistency } \\
\text { between SC would be } \\
\text { important diagnostic } \\
\text { for spatial } \\
\text { boundaries. }\end{array}$ \\
\hline 5 & $\begin{array}{l}\text { Ion } \\
\text { anisotropy }\end{array}$ & EIS ions & $\begin{array}{l}25-500 \mathrm{keV} \\
\text { protons }\end{array}$ & $\begin{array}{l}\text { Indexed to } \\
0-5 \text { (TBR) }\end{array}$ & $\begin{array}{l}\text { Ratio of selectred } \\
\text { look directions for } \\
\text { common energy } \\
\text { ranges. Maximum } \\
\text { values over } 1 / 2 \mathrm{SC} \\
\text { spin }\end{array}$ & $\begin{array}{l}\text { Remote sensing of } \\
\text { incoming boundaries. }\end{array}$ \\
\hline
\end{tabular}

the MET time is recorded. For each record the "accumulation time" must be reported. For each SSD detector (12 for FEEPS and 12 out of the possible 24 for EIS) the "Livetime" or "Deadtime" are recorded for performing corrections to the detector counting rates. Also accompanying each data record here is magnetic field data in spacecraft coordinates. EIS and FEEPS data are joined together to create a "Fast Ion" product. In order to do this joining, "Transfer files" are generated from the EIS data, which is then used with the FEEPS process. What this means is that the EIS Level-2 is created first, followed by the creation of the FEEPS Level-2 data. The List of Level 2 data products are listed below.

\section{L2-FEEPS-Electron- Burst}

2. L2-FEEPS-Integral-Electron-Burst

3. L2-FEEPS-Electron- Survey

4. L2-Fast-Ion-Burst

5. L2-Fast-Ion-Survey 


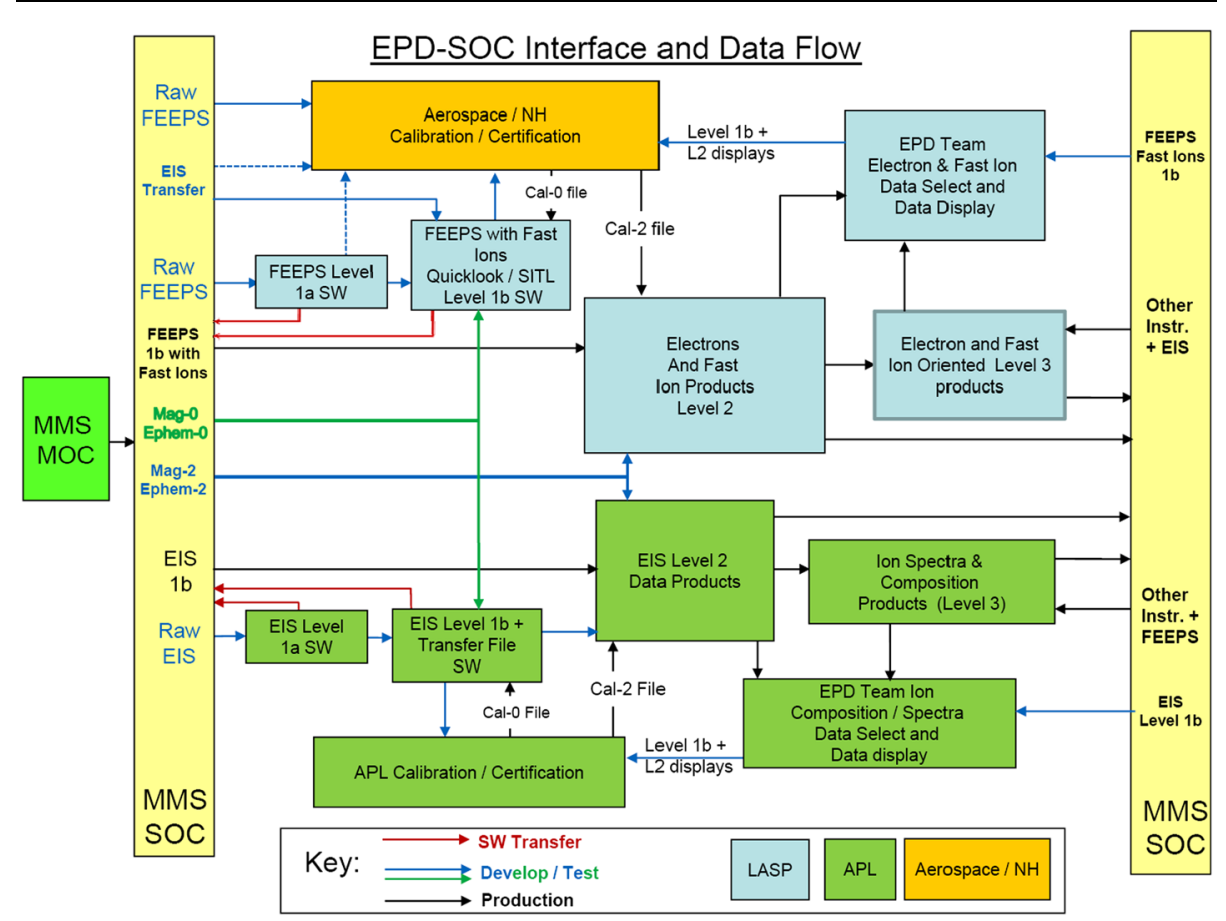

Fig. 31 EPD data processing flow diagram

6. L2-EIS-PhTOF-Ion-Burst

7. L2-EIS-E $\times$ TOF-Ion-Burst

8. L2-EIS-PhTOF-Ion-Survey

9. L2-EIS-E $\times$ TOF-Ion-Survey

10. L2-EIS-Transfer-burst

11. L2-EIS-Transfer-survey

12. L2-EIS-Electron-Energy-Burst (diagnostic)

13. L2-EIS-Electron-Energy-Survey (diagnostic)

EPD Level-3 Data (Preliminary) This level contains a variety of products that are, to this date, not fully defined. These products require: (1) Extensive calculations requiring hands-on certification, or (2) the joining of multiple data sets. Two specific data products are already known to be required: (i) A product that converts the rough all sky electron images into high resolution all sky electron images via a field-of-view deconvolution process with the FEEPS sensors, and (ii) A product that generates full ion spectra by combining the EIS TOF $\times \mathrm{E}$ and TOF $\times$ PH data sets. Other Level 3 products include moments; spectra derived by combining some combinations of EPD with HPCA and FPI; Moments similarly derived by combining some combinations of EPD with HPCA and FPI, etc.

Open Access This article is distributed under the terms of the Creative Commons Attribution License which permits any use, distribution, and reproduction in any medium, provided the original author(s) and the source are credited. 


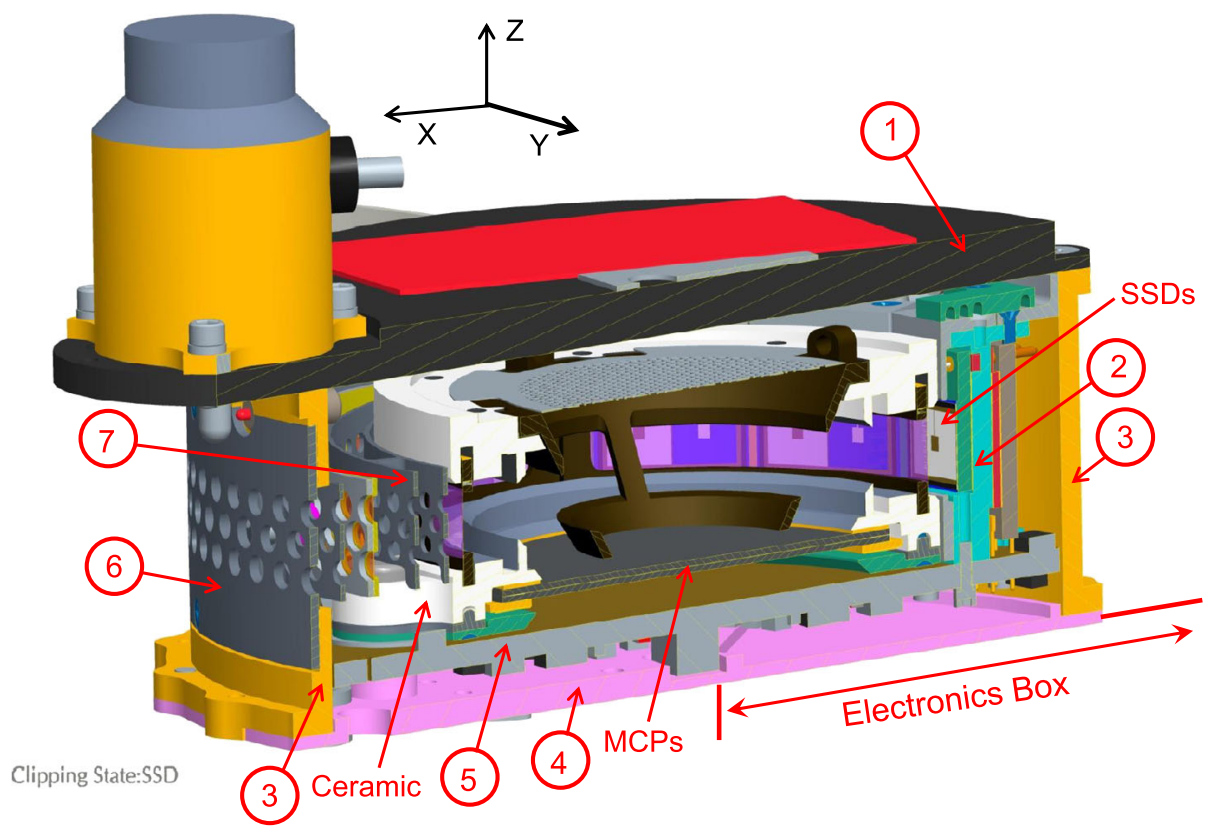

Fig. 32 Cross section of one of the EIS sensors showing the shielding configuration for both the SSD and the MCP sensors (shown is actually the JEDI version with holes rather than slots in the collimator). See the text in the Appendix for a discussion of this figure

\section{Appendix: EIS Detailed Specifications}

Provide here are quantitative specifications for each EIS instrument.

\section{A.1 EIS Instrument}

This list refers to each of 4 instruments. Below we provide geometric factors, but these do not include efficiencies (see Fig. 22). For other measurement characteristics of the EIS instrument (energy and species responses and resolutions, etc.) see Table 3.

\footnotetext{
Total Mass:

$2.2 \mathrm{~kg}$ (incl: EMI, thermal, heaters, internal harness)

Operational Power:

$2.0 \mathrm{~W}$

Volume:

$23.3 \times 16.9 \times 12.8 \mathrm{~cm}$ (box holding instrument; door stowed)

Data Rate:

Field of View:

Burst: 12 kbps; Fast Survey: 0.67 kbps

Angle resolution:

$160 \times 12$ degrees

Total TOF $\times$ PH G:

$26.7^{\circ} \times 12^{\circ}$

Pixel TOF $\times$ PH G:

$0.03 \mathrm{~cm}^{2} \mathrm{sr}$

$0.0051 \mathrm{~cm}^{2} \mathrm{sr}$

Total Energy G:

$0.012 \mathrm{~cm}^{2} \mathrm{sr}$ (ion or electron, not both)

Large Pixel Energy G: $\quad 0.002 \mathrm{~cm}^{2} \mathrm{sr}$

Small Pixel Energy G: $\quad 0.0001 \mathrm{~cm}^{2} \mathrm{sr}$ (ion or electron, not both)
} 


\begin{tabular}{|c|c|c|c|c|c|c|c|c|c|c|c|c|}
\hline & \multicolumn{4}{|c|}{ TOF $\times \mathrm{PH}$} & \multicolumn{4}{|c|}{ TOF $\times \mathrm{E}$ and lons } & \multicolumn{4}{|c|}{ Electrons } \\
\hline & Degrees & Unit-X & Unit- $Y$ & Unit-Z & Degrees & Unit-X & Unit- Y & Unit-Z & Degrees & Unit-X & Unit- $Y$ & Unit-Z \\
\hline vo & 72.18 & 0.306 & -0.952 & 0.000 & 72.18 & 0.306 & -0.952 & 0.000 & 61.12 & 0.483 & -0.876 & 0.000 \\
\hline v1 & 45.56 & 0.700 & $\mid-0.714$ & 0.000 & 45.56 & 0.700 & -0.714 & 0.000 & 34.5 & 0.824 & -0.566 & 0.000 \\
\hline v2 & 18.84 & 0.946 & -0.323 & 0.000 & 18.84 & 0.946 & -0.323 & 0.000 & 7.78 & 0.991 & -0.135 & 0.000 \\
\hline v3 & -7.78 & 0.991 & 0.135 & 0.000 & -7.78 & 0.991 & 0.135 & 0.000 & -18.84 & 0.946 & 0.323 & 0.000 \\
\hline v4 & -34.5 & 0.824 & 0.566 & 0.000 & -34.5 & 0.824 & 0.566 & 0.000 & -45.56 & 0.700 & 0.714 & 0.000 \\
\hline v5 & -61.12 & 0.483 & 0.876 & 0.000 & -61.12 & 0.483 & 0.876 & 0.000 & -72.18 & 0.306 & 0.952 & 0.000 \\
\hline
\end{tabular}

Fig. 33 EIS look directions for the three classes of data products. Look directions v2 and v3 have various levels of sun-shield blockages that have not, in this table, been yet accommodated. The off-color rows for TOF $\times \mathrm{E}$ and electrons are blocked most substantially

\section{A.2 EIS Viewing}

The coordinate system for each EIS sensor is shown in Figs. 16, 17 and 32. The coordinate system for each EIS sensor has its X-axis pointing parallel to the center of its $160^{\circ} \times 12^{\circ}$ field-of-view, with the $160^{\circ}$ direction contained within the $\mathrm{X}-\mathrm{Y}$ plane, with the positive $Y$ Axis closest to the V5 direction. In Fig. 33 the central direction of the center of each $\mathrm{TOF} \times \mathrm{PH}$, Ion-SSD, and Electron-SSD pixel is given as the angle from the $\mathrm{X}$-axis within the $\mathrm{X}-\mathrm{Y}$ plane, with positive angles towards the $-\mathrm{Y}$ axis (also toward the direction that has been designated the "V0" direction; we realize that it is unusual to have positive angles towards the $-\mathrm{Y}$ axis rather than the $+\mathrm{Y}$ axis). To the right of each angle in Fig. 33 is the unit vector of the view direction in the instrument coordinate system. Views V2 and V3 have obstruction from the shielding needed to keep the sun out (Fig. 10), and that obstruction has not been yet folded into the table (the off-color rows are the views that are substantially blocked). The spacecraft coordinate system is shown in Figs. 2 and 8. Below we provide the transformation matrix that converts the coordinate system of each of the EIS sensors into the MMS spacecraft coordinate system. To transform an EIS coordinate vector ( $\left.\mathbf{V}_{\text {EIS }}\right)$ into the spacecraft frame $\left(\mathbf{V}_{\mathbf{S C}}\right)$, one performs the operation: $\mathbf{V}_{\mathbf{S C}}=\mathbf{T}(\mathbf{E I S}) \bullet \mathbf{V}_{\text {EIS }}$ where:

$$
\mathbf{T}(\mathbf{E I S})=\left[\begin{array}{ccc}
-1 / \sqrt{2} & 1 / \sqrt{2} & 0 \\
0 & 0 & 1 \\
1 / \sqrt{2} & 1 / \sqrt{2} & 0
\end{array}\right]
$$

\section{A.3 Electronics Box}

$\begin{array}{ll}\text { Mass: } & 1.4 \mathrm{~kg} \\ \text { Shield thickness: } & 100 \text { mils } \\ \text { Shield material: } & \text { Aluminum } \\ \text { Size: } & 15.9 \times 20.7 \times 9.3 \mathrm{~cm} \text { (including feet \& EMI shield) } \\ \text { Volume: } & 1.06 \text { Liters (excluding structure, EMI shield, and feet) } \\ \text { Operational Power: } & 1.53 \text { Watts (Does not include make-up SC heaters) }\end{array}$


A.4 Sensor

\section{A.4.1 Sensor Bulk Properties and External Structure}

$\begin{array}{ll}\text { Mass: } & 0.5 \mathrm{~kg} \\ \text { Volume: } & 196.35 \mathrm{~cm}^{3} \\ \text { Shield thickness: } & \text { See Section A.5 } \\ \text { Shield material: } & \text { See Section A.5 } \\ \text { Radiation tolerance: } & \text { All parts > 100 Krad (EEPROM in radpack) } \\ \text { Operational Power } & \text { 0.33 Watts }\end{array}$

\section{A.4.2 Collimator (Structure, Distances, Thicknesses, Slot Sizes, etc)}

The collimator (Figs. 10 and 19) is made of Aluminum. In the list below, "radius" is the radial position of the outer surface of each blade from the center of the cylindrical TOF chamber. The slots in the blades are linearly graduated according to that radial position. Finally, all lines that pass through the centers of the slots as one moves from blade to blade also pass through the single point that is the symmetry position of the cylindrical TOF chamber (in cylindrical coordinates the symmetry point not only in " $r$ " but in " $z$ " as well; see Fig. 10). The slots are characterized with a width (W; in the instrument "XY" plane) and a Height ( $\mathrm{H}$ : in the instrument " $\mathrm{Z}$ " direction). Blade 1 is the outer blade.

$\begin{array}{lllll}\text { Blade 1: } & \text { Radius: } 4.99 \mathrm{~cm} ; & \text { Thickness: } 0.101 \mathrm{~cm} ; & \text { W: } 0.28 \mathrm{~cm} & \mathrm{H}: 0.37 \mathrm{~cm} \\ \text { Blade 2: } & \text { Radius: } 4.60 \mathrm{~cm} ; & \text { Thickness: } 0.050 \mathrm{~cm} ; & \text { W: } 0.31 \mathrm{~cm} & \mathrm{H}: 0.43 \mathrm{~cm} \\ \text { Blade 3 (foil): } & \text { Radius: } 4.17 \mathrm{~cm} ; & \text { Thickness: } 0.051 \mathrm{~cm} ; & \text { W: } 0.34 \mathrm{~cm} & \mathrm{H}: 0.48 \mathrm{~cm} \\ \text { Blade 4: } & \text { Radius: } 3.63 \mathrm{~cm} ; & \text { Thickness: } 0.051 \mathrm{~cm} ; & \text { W: } 0.38 \mathrm{~cm} & \mathrm{H}: 0.55 \mathrm{~cm} \\ \text { Blade 5: } & \text { Radius: } 3.25 \mathrm{~cm} ; & \text { Thickness: } 0.051 \mathrm{~cm} ; & \text { W: } 0.41 \mathrm{~cm} & \mathrm{H}: 0.61 \mathrm{~cm}\end{array}$

\section{A.4.3 Foils (See Fig. 19)}

In the list below, "radius" is the radial position of the foil from the center of the cylindrical TOF chamber

Collimator foil: $\quad$ Radius: $4.166 \mathrm{~cm} ; 350 \AA \mathrm{Al}$

Collimator grid: 70 line/inch Stainless Steel, $90 \%$ transmission normal incidence

Start foil: $\quad$ Radius: $3.0 \mathrm{~cm}$; $50 \AA$ Carbon, $350 \AA$ polyimide, $50 \AA$ Carbon

Stop foil: $\quad$ Radius: $3.0 \mathrm{~cm}$; Start foil $+200 \AA \mathrm{Al}$

Start/Stop Grids: 70 lines/inch Ni, $90 \%$ transmission normal incidence

\section{A.4.4 TOF Chamber (see Fig. 12)}

In the list below, "radius" is the radial position of the outer surface of the chamber from the center of the chamber.

Entrance Window: Height: $0.6 \mathrm{~cm}$

Radius of window: $3.0 \mathrm{~cm}$

Chamber diameter: $\quad 6.0 \mathrm{~cm}$ 


\section{A.4.5 MCP Sensors (See Fig. 18)}

The MCP comprises 2 circular Burle MCP plates in the chevron configuration with a small gap between them and operated with high voltages between 1900 and 2400 volts over the life-time of the plates.

$\begin{array}{ll}\text { Diameters: } & 5 \mathrm{~cm} ; 4 \mathrm{~cm} \text { quality diameter } \\ \text { Plate thickness: } & 0.06 \mathrm{~cm} \\ \text { Pore size: } & 10 \mathrm{microns} \\ \text { Pore pitch: } & 12 \mu \mathrm{m} \\ \text { Bias angle: } & 12^{\circ} \\ \text { Stack gain: } & \text { Several } \times 10^{6}\end{array}$

\section{A.4.6 SSDs and SSD Arrays (see Figs. 18 and 20)}

Each EIS comprises 6 SSD's with 4 pixels each manufactured by Canberra. The electrical signal from the small pixels is measured using a "channel" that runs across the face of the large pixel (Fig. 20) that can contribute a small degraded contaminating signal to the large pixel response. The SSD is mounted on printed circuit board (all but hidden in Fig. 20), which in turn is mounted on the Aluminum "hangers." In the list below, the word "radius" refers to the radial position with respect to the center of the TOF chamber.

$\begin{array}{ll}\text { Radius of SSD array: } & \text { Surface facing particles: } 3.38 \mathrm{~cm} \\ \text { Thickess: } & 500 \text { microns }(0.05 \mathrm{~mm}) \\ \text { Deadlayers: } & \text { Roughly } 50 \mathrm{~nm} \text { as measured by EIS team } \\ \text { Size: } & 14.7 \times 8.5 \mathrm{~mm} \\ \text { Large pixel size: } & 6.20 \times 6.45 \mathrm{~mm} \text {; Area }=40 \mathrm{~mm}^{2} \\ \text { Small pixel size: } & 1.28 \times 1.55 \mathrm{~mm} \text {; Area }=2 \mathrm{~mm}^{2} \\ \text { Electron pixel flashing: } & 2 \text { microns Al } \\ \text { Channel size: } & 0.2 \times 2.3 \mathrm{~mm} \text {; Area }=0.46 \mathrm{~mm}^{2}\end{array}$

\section{A.5 Sensor Shielding}

Figure 32 shows a cross section of the EIS proxy (JEDI) with the various elements labeled that provide the shielding for the SSD and for the MCP (Note that the one observable difference between JEDI and EIS is the hole-structure of the collimator). This cut through the sensor is rotated somewhat away from the symmetry axis of the sensor (contrary to what is shown in Fig. 17). There is a menagerie of structures that provide partial protection to these sensors, but labeled here are the primary structures that are specifically designed to provide shielding. Item (1) is 200 mils of Al providing shielding of $0.86 \mathrm{gm} / \mathrm{cm}^{2}$. The cylindrical can (Item (3)) is 100 mils of Al providing shielding of $0.43 \mathrm{gm} / \mathrm{cm}^{2}$. The SSD hangers (Item (2)) provide an additional 100 mils of $\mathrm{Al}$, or $0.43 \mathrm{gm} / \mathrm{cm}^{2}$ shielding to the backs of the SSD's for a total to the backs of the SSD of $0.86 \mathrm{gm} / \mathrm{cm}^{2}$. A combination of Items (4) and (5) (100 mils $\mathrm{Al}$ each) and the very well shielded electronics box, provides a minimum of 200 mils of $\mathrm{Al}$ or $0.86 \mathrm{gm} / \mathrm{cm}^{2}$, to the bottom of the sensor can. From the direction of the sensor field of view to the left, those particles that do not enter through the sensor holes are blocked (Items (6) and (7)) with a total of 120 mils of $\mathrm{Al}$ distributed within the 5 blades of the collimator (with thickness of 40, 20, 20, 20, and 20 mils of $\mathrm{Al}$ ) for a total of $0.52 \mathrm{gm} / \mathrm{cm}^{2}$. We note that the amount of shielding is very modest such that EIS will not make the kind of 
clean electron measurements that FEEPS will. It is the coincident circuitry used for all of the quality ion measurements that make this shielding configuration work well for EIS ion measurements for all MMS target regions.

\section{References}

V. Angelopoulos, A. Runov, X.-Z. Zhou, D.L. Turner, S.A. Kiehas, S.-S. Li, I. Shinohara, Electromagnetic energy conversion at reconnection fronts. Science 341(6153), 1478 (2013). doi:10.1126/science. 1236992

D.N. Baker et al., Timing of magnetic reconnection initiation during a global magnetospheric substorm onset. Geophys. Res. Lett. 29(24), 2190 (2002). doi:10.1029/2002GL015539

D.N. Baker, T.I. Pulkkinen, V. Angelopoulos, W. Baumjohann, R.L. McPherron, Neutral line model of substorms: past results and present view. J. Geophys. Res. 101(A6), 12975-13010 (1996). doi:10.1029/ 95JA03753

D.N. Baker et al. MMS Data Management, this issue

J. Birn, M. Hesse, Particle acceleration in the dynamic magnetotail: orbits in self-consistent three-dimensional MHD fields. J. Geophys. Res. 99(A1), 109-119 (1994). doi:10.1029/93JA02284

J. Birn, M. Hesse, R. Nakamura, S. Zaharia, Particle acceleration in dipolarization events. J. Geophys. Res. 118, 1960-1971 (2013). doi:10.1002/jgra.50132

J.B. Blake et al., The Flys Eye Energetic Particle Spectrometer (FEEPS) contribution to the Energetic Particle Detector (EPD) investigation of the Magnetospheric Magnetoscale (MMS) Mission, this issue

P.K. Browning, G.E. Vekstein, Particle acceleration at a Z-type reconnection site with a parallel magnetic field. J. Geophys. Res. 106(A9), 18677 (2001). doi:10.1029/2001JA900014

J.L. Burch et al., MMS Overview and Science Objectives, this issue

S.P. Christon, D.J. Williams, D.G. Mitchell, C.Y. Huang, L.A. Frank, Spectral characteristics of plasma sheet ion and electron populations during disturbed geomagnetic conditions. J. Geophys. Res. 96(A1), 1-22 (1991). doi:10.1029/90JA01633

J.E. Connerney et al., Juno magnetometer observations in Earth's magnetosphere, in American Geophysical Union Fall Meeting, SM21E-04, San Francisco, 10 December (2013)

D.C. Delcourt, J.A. Sauvaud, A. Pedersen, Dynamics of single-particle orbits during substorm expansion phase. J. Geophys. Res. 95(A12), 20853-20865 (1990). doi:10.1029/JA095iA12p20853

J.F. Drake, M. Swisdak, C. Cattell, M.A. Shay, B.N. Rogers, A. Zeiler, Formation of electron holes and particle energization during magnetic reconnection. Science 299, 873 (2003). doi:10.1126/science.1080333

J.F. Drake, M. Swisdak, W. Thongthai, M.A. Shay, Production of energetic electrons during magnetic reconnection. Phys. Rev. Lett. 94, 095001 (2005). doi:10.1103/PhysRevLett.94.095001

J. Egedal, A. Lê, Y. Zhu, W. Daughton, M. Øieroset, T. Phan, R.P. Lin, J.P. Eastwood, Cause of super-thermal electron heating during magnetotail reconnection. Geophys. Res. Lett. 37, L10102 (2010). doi:10.1029/ 2010GL043487

M. Fujimoto, M. Nakamura, Acceleration of heavy ions in the magnetotail reconnection layer. Geophys. Res. Lett. 21, 2955 (1994). doi:10.1029/94GL02102

E.E. Grigorenko, M. Hoshino, M. Hirai, T. Mukai, L.M. Zelenyi, "Geography" of ion acceleration in the magnetotail: X-line versus current sheet effects. J. Geophys. Res. 114, A03203 (2009). doi:10.1029/ 2008JA013811

E.E. Grigorenko, L.M. Zelenyi, M.S. Dolgonosov, A.V. Artemiev, C.J. Owen, J.-A. Sauvaud, M. Hoshino, M. Hirai, Non-adiabatic ion acceleration in the Earth magnetotail and its various manifestations in the plasma sheet boundary layer. Space Sci. Rev. 164, 133-181 (2011). doi:10.1007/s11214-011-9858-9

J.R. Hayes, The Scalable Configurable Instrument Processor, Military and Aerospace Programmable Logic Devices (MAPLD) Conference (2005)

M. Hoshino, T. Mukai, T. Terasawa, I. Shinohara, Suprathermal electron acceleration in magnetic reconnection. J. Geophys. Res. 106(A11), 25979-25997 (2001). doi:10.1029/2001JA900052

M. Hoshino, Electron surfing acceleration in magnetic reconnection. J. Geophys. Res. 110, A10215 (2005). doi:10.1029/2005JA011229

S.M. Krimigis et al., Magnetosphere imaging instrument (MIMI) on the Cassini mission to Saturn/Titan. Space Sci. Rev. 114(1-4), 233-329 (2004). doi:10.1007/s11214-004-1410-8

M.M. Kuznetsova, M. Hesse, D. Winske, Ion dynamics in a hybrid simulation of magnetotail reconnection. J. Geophys. Res. 101(A12), 27351-27373 (1996). doi:10.1029/96JA02622

R.P. Lin, H.S. Hudson, 10-100 keV electron acceleration and emission from solar flares. Sol. Phys. 17, 412 (1971). doi:10.1007/BF00150045

W.H. Matthaeus, J.J. Ambrosiano, M.L. Goldstein, Particle acceleration by turbulent magnetohydrodynamic reconnection. Phys. Rev. Lett. 53, 1449 (1984). doi:10.1103/PhysRevLett.53.1449 
B.H. Mauk, Quantitative modeling of the "convection surge" mechanism of ion acceleration. J. Geophys. Res. 91(A12), 13423-13431 (1986). doi:10.1029/JA091iA12p13423

B.H. Mauk, Generation of macroscopic magnetic-field-aligned electric fields by the convection surge ion acceleration mechanism. J. Geophys. Res. 94(A7), 8911-8920 (1989). doi:10.1029/JA094iA07p08911

B.H. Mauk et al., The Jupiter energetic particle detector instrument (JEDI) investigation for the Juno mission. Space Sci. Rev. (2013). doi:10.1007/s11214-013-0025-3

R.E. McNutt et al., The Pluto energetic particle spectrometer on the New Horizons mission. Space Sci. Rev. 140(1-4), P315 (2008). doi:10.1007/s11214-0089436-y

D.G. Mitchell, F. Kutchko, D.J. Williams, T.E. Eastman, L.A. Frank, C.T. Russell, An extended study of the low-latitude boundary layer on the dawn and dusk flanks of the magnetosphere. J. Geophys. Res. 92(A7), 7394-7404 (1987). doi:10.1029/JA092iA07p07394

D.G. Mitchell, P.C. Brandt, E.C. Roelof, D.C. Hamilton, K.C. Retterer, S. Mende, Global imaging of $\mathrm{O}^{+}$ from IMAGE/HENA. Space Sci. Rev. 109, 63-75 (2003). doi:10.1023/B:SPAC.0000007513.55076.00

D.G. Mitchell et al., Radiation Belt Storm Probes Ion Composition Experiment (RBSPICE). Space Sci. Rev. 179(1-4), 263 (2013). doi:10.1007/s11214-013-9965-X

R.W. McEntire, E.P. Keath, D.E. Fort, A.T.Y. Lui, S.M. Krimigis, The medium energy particle analyzer on the AMPTEE CCE spacecraft, geoscience and remote sensing. IEEE Trans. Geosci. Remote Sens. GE23(3), 230 (1985). doi:10.1109/TGRS.1985.289518

M. Nosé, A.T.Y. Lui, S. Ohtani, B.H. Mauk, R.W. McEntire, D.J. Williams, T. Mukai, K. Yumoto, Acceleration of oxygen ions of ionospheric origin in the near-Earth magnetotail during substorms. J. Geophys. Res. 105(A4), 7669-7677 (2000). doi:10.1029/1999JA000318

M. Øieroset, R.P. Lin, T.D. Phan, D.E. Larson, S.D. Bale, Evidence for electron acceleration up to $\sim 300 \mathrm{keV}$ in the magnetic reconnection diffusion region of Earth's magnetotail. Phys. Rev. Lett. (2002). doi:10. 1103/PhysRevLett.89.195001

C.J. Owen, R. Mist, Distant plasma sheet ion distributions during reconnection. Geophys. Res. Lett. 28(14), 2771 (2001). doi:10.1029/2001GL013032

N.P. Paschalidis, A family of analog and mixed signal VLSI ASICs for NASA science missions. Acta Astronaut. 59, 974-980 (2006). doi:10.1016/j.actaastro.2005.07.044

N.P. Paschalidis et al., A CMOS time of flight system on a chip for spacecraft instrumentation. IEEE Trans. Nucl. Sci. 49, 1156-1163 (2002). doi:10.1016/j.actaastro.2005.07.044

R.J. Pellinen, W.J. Heikkila, Energization of charged particles to high energies by an induced substorm electric field within the magnetotail. J. Geophys. Res. 83(A4), 1544-1550 (1978). doi:10.1029/ JA083iA04p01544

P.L. Pritchett, Externally driven magnetic reconnection in the presence of a normal magnetic field. J. Geophys. Res. 110, A05209 (2005). doi:10.11029/2004JA010948

A. Runov, V. Angelopoulos, M.I. Sitnov, V.A. Sergeev, J. Bonnell, J.P. McFadden, D. Larson, K.-H. Glassmeier, U. Auster, THEMIS observations of an earthward-propagating dipolarization front. Geophys. Res. Lett. 36, L14106 (2009). doi:10.1029/2009GL038980

D. Sachsenweger, M. Scholer, E. Möbius, Test particle acceleration in a magnetotail reconnection configuration. Geophys. Res. Lett. 16(9), 1027 (1989). doi:10.1029/GL016i009p01027

E.R. Sánchez, B.H. Mauk Ching, I. Meng, Adiabatic vs. non-adiabatic particle distributions during convection surges. Geophys. Res. Lett. 20, 177 (1993). doi:10.1029/93GL00237

T. Sato, H. Matsumoto, K. Nagai, Particle acceleration in time-developing magnetic reconnection process. J. Geophys. Res. 87(A8), 6089-6097 (1982). doi:10.1029/JA087iA08p06089

M. Scholer, P.W. Daly, G. Paschmann, T.A. Fritz, Field line topology determined by energetic particles during a possible magnetopause reconnection event. J. Geophys. Res. 87(A8), 6073-6080 (1982). doi:10.1029/ JA087iA08p06073

M. Scholer, B. Klecker, D. Hovestatd, G. Gloeckler, F.M. Ipavich, A.B. Galvin, D.N. Baker, B.T. Tsurutani, Energetic ion and electron beams at the plasma-sheet boundary in the distant tail, in Magnetotail Physics (A88-46526 19-46), ed. by A.T.Y. Lui (Johns Hopkins University Press, Baltimore, 1987), pp. 245-249

M.I. Sitnov, M. Swisdak, A.V. Divin, Dipolarization fronts as a signature of transient reconnection in the magnetotail. J. Geophys. Res. 114, A04202 (2009). doi:10.1029/2008JA013980

D.J. Williams, R.W. McEntire, S. Jaskulek, B. Wilken, The Galileo energetic particle detector. Space Sci. Rev. 60, 385 (1992). doi:10.1007/978-94-011-2512-3_16

D.J. Williams, R.W. McEntire, C. Schlemm II., A.T.Y. Lui, G. Gloeckler, S.P. Christon, F. Gliem, Geotail energetic particles and ion composition instrument. J. Geomagn. Geoelectr. 46(1), 39-57 (1994). doi: 10. 5636/jgg.46.39 